\title{
T-Dagum: A Way of Generalizing Dagum Distribution Using Lomax Quantile Function
}

\author{
Matthew I. Ekum (D), Muminu O. Adamu, and Eno E. Akarawak \\ Department of Mathematics, University of Lagos, Akoka, Lagos, Nigeria \\ Correspondence should be addressed to Matthew I. Ekum; matekum@yahoo.com
}

Received 16 November 2019; Revised 25 February 2020; Accepted 23 March 2020; Published 24 April 2020

Academic Editor: Ramón M. Rodríguez-Dagnino

Copyright (C) 2020 Matthew I. Ekum et al. This is an open access article distributed under the Creative Commons Attribution License, which permits unrestricted use, distribution, and reproduction in any medium, provided the original work is properly cited.

\begin{abstract}
Recently, different distributions have been generalized using the $T-R\{Y\}$ framework but the possibility of using Dagum distribution has not been assessed. The $T-R\{Y\}$ combines three distributions, with one as a baseline distribution, with the strength of each distribution combined to produce greater effect on the new generated distribution. The new generated distributions would have more parameters but would have high flexibility in handling bimodality in datasets and it is a weighted hazard function of the baseline distribution. This paper therefore generalized the Dagum distribution using the quantile function of Lomax distribution. A member of $T$-Dagum class of distribution called exponentiated-exponential-Dagum \{Lomax (EEDL) distribution was proposed. The distribution will be useful in survival analysis and reliability studies. Different characterizations of the distribution are derived, such as the asymptotes, stochastic ordering, stress-strength analysis, moment, Shannon entropy, and quantile function. Simulated and real data are used and compared favourably with existing distributions in the literature.
\end{abstract}

\section{Introduction}

The quality of the result of a statistical model depends so much on the fitness of the assumed probability distribution to the data. Thus, significant effort has been made in developing different families of probability distributions along with their relevant statistical methods [1].

However, there are still many important real problems where any of the existing standard and newly developed distributions do not fit the data appropriately, especially in the areas of finance, engineering, medicine, and environmental hazards. The Dagum distribution is one of the most important distributions in modeling income and wealth distribution, especially personal income, and is mostly associated with the study of income distribution [2]. It is related to the Gini index (see [3]); it is not the only threeparameter distribution used to model income distribution but it is often most appropriate [4].

The following Dagum distributions have been proposed: beta-Dagum distribution [5], Mc-Dagum Distribution [6], weighted Dagum distribution [7], gamma-Dagum distribution [8], exponentiated Kumaraswamy-Dagum distribution [9], extended Dagum distribution [10], transmuted Dagum distribution [11], Dagum-Poisson distribution [12], exponentiated generalized exponential Dagum distribution [13], and power log-Dagum distribution [14].

Johnson et al. [15] asserted that the use of four-parameter distributions should be sufficient for most practical purposes and that at least three parameters are needed to model any real data, but they doubted any noticeable improvement arising from, including a fifth or sixth parameter. However, we were motivated by the work of [13], with a sixparameter distribution. Their six-parameter distribution performed better than its submodels with fewer parameters. Other authors have also demonstrated and showed that distributions with more parameters have greater flexibility of modelling reliability and survival data than their submodels with fewer parameters, thereby proving Johnson et al. [15] wrong in their statement. Aljarrah et al. [16] mentioned that adding the fifth parameter to the Normal-Weibull\{Cauchy\} distribution improved the fit of the model to the data with an increase of more than 22 points in the log-likelihood value. Several works by the following authors, Paranaíba et al. [17]; Cordeiro and Lemonte [18]; Domma and Condino [5]; 
Oluyede et al. [8]; Silva et al. [10]; and Bakouch et al. [14], further supported the work of Nasiru et al. [13].

The motivation of this work is that the Dagum distribution despite being one of the most important and appropriate distributions in modelling income and wealth, and its relationship with Gini Index, is not being generalized via the $T-R\{Y\}$ framework. The $T-R\{Y\}$ framework is a combination of 3 distributions, $T, R$, and $Y$, where the quantile function of $Y$ is used as a frame to hold the cdf of $R$, which is being transformed by $T$, with some parameters of each distribution having effect on the newly formed distribution. One major importance of providing new distribution through the quantile function of an existing distribution is that the newly formed distribution has the tendency of having higher flexibility in handling bimodality in datasets and it is a weighted hazard function of the baseline distribution (Dagum distribution in this case). For more detailed informations on the importance of using this method, $T-R\{Y\}$, see Aljarrah et al. [16]; Alzaatreh et al. [19, 20]; Zubair et al. [21]; and Famoye et al. [22]. Also, for detailed knowledge of Dagum distribution, see Bandourian et al. [4]; Kleiber and Kotz [2]; Kleiber [3]; Domma and Condino [5]; Oluyede and Rajasooriya [6]; Oluyede and Ye [7]; Oluyede et al. [8]; Huang and Oluyede [9]; Silva et al. [10]; Shahzad and Asghar [11]; Oluyede et al. [12]; Nasiru et al. [13]; and Bakouch et al. [14].

Thus, in this study, a new generalization of the Dagum distribution named that the $T$-Dagum $\{Y\}$ family is generated and a member of this family, the exponentiated-exponentialDagum\{Lomax (EEDL) distribution with six parameters, is proposed and its properties are studied. This proposed distribution will not only take into consideration high flexibility in the shape and scale parameters but also takes care of skewness (right and left), kurtosis and tail variation, and sometimes can be stable for some parameter values.

The rest of the article is organized as follows. In Section 2, the proposed distribution was derived along with some of its characterizations, and the parameters of the proposed distribution were estimated using maximum likelihood estimation (MLE). A simulation study to assess the stability and performance of the parameter estimates was carried out. This is followed by the application of the new model demonstrated using two real datasets, and finally conclusion is given based on the simulation study and the real applications.

\section{Proposed $T$-Dagum $\{\boldsymbol{Y}\}$ Class}

The beta-generated family defined as $T-X$ family by [23] was extended by [24] to $T-X\{W\}$ family, and further extension was made by [16] to the $T-X\{W\}$ by making $W[F(x)]$ to be the quantile function of a random variable $Y$ and defined the $T-X\{Y\}$ family as

$$
G(x)=\int_{a}^{Q_{Y} F(x)} r(t) \mathrm{d} t=R\left\{Q_{Y}[F(x)]\right\} .
$$

The $T-X\{W\}$ in (1) was redefined by Alzaatreh et al. [19] as $T-R\{Y\}$. They gave the unified definition of $T-R\{Y\}$ family. The cdf of the $T-R\{Y\}$ family is defined by

$$
\begin{aligned}
F_{X}(x) & =\int_{a}^{Q_{Y}\left[F_{R}(x)\right]} f_{T}(t) \mathrm{d} t=P\left\{T \leq Q_{Y}\left[F_{R}(x)\right]\right\} \\
& =F_{T}\left\{Q_{Y}\left[F_{R}(x)\right]\right\},
\end{aligned}
$$

where $f_{T}(t)$ is the pdf of a random variable $T, Q_{Y}(\cdot)$ is the quantile function of a random variable $Y$ and $F_{R}(x)$ is the cdf of a random variable $R . Q_{Y}\left[F_{R}(x)\right]$ is differentiable and monotonically nondecreasing. It is necessary that and $f_{T}(t)$ have the same support.

The pdf corresponding to the $\mathrm{cdf}$ in (2) is given by

$$
f_{X}(x)=f_{R}(x) \frac{f_{T}\left\{Q_{Y}\left[F_{R}(x)\right]\right\}}{f_{Y}\left\{Q_{Y}\left[F_{R}(x)\right]\right\}} .
$$

In the literature, many authors have used this $T-\mathrm{R}\{\mathrm{Y}\}$ framework to develop probability distributions, such as Aljarrah et al. [16]; Alzatraah et al. [19, 20]; Nasir et al. [25, 26]; Jamal et al. [27, 28]; Zubair et al. [21]; Famoye et al. [22]; and Jamal and Nasir [29]. None of these authors has generalized Dagum distribution using this framework.

In this research, we let $R$ be a random variable that follows Dagum distribution with cdf, and $F_{R}(x)$ is given by

$$
F_{R}(x)=\left[1+\left(\frac{x}{v}\right)^{-u}\right]^{-q} .
$$

The cdf of the $T$-Dagum $\{Y\}$ family is thus defined by putting equation (4) in (2) to have

$$
F_{X}(x)=F_{T}\left\{Q_{Y}\left[\left[1+\left(\frac{x}{v}\right)^{-u}\right]^{-q}\right]\right\} .
$$

Equation (5) is the cdf, and $F_{X}(x)$ of the proposed $T$-Dagum $\{Y\}$ (or simply $T-D\{Y\}$ ) class of distribution. Let the pdf of Dagum distribution be given as

$$
f_{R}(x)=\frac{u q}{x}\left\{\frac{(x / v)^{u q}}{\left[1+(x / v)^{u}\right]^{q+1}}\right\}, \quad x>0 ; u, q, v>0 .
$$

From equation (3), the corresponding pdf to equation (5) is given by

$$
f_{X}(x)=\frac{u q}{x}\left\{\frac{(x / v)^{u q}}{\left[1+(x / v)^{u}\right]^{q+1}}\right\} \times \frac{f_{T}\left\{Q_{Y}\left[\left[1+(x / v)^{-u}\right]^{-q}\right]\right\}}{f_{Y}\left\{Q_{Y}\left[\left[1+(x / v)^{-u}\right]^{-q}\right]\right.} .
$$

Remark 1. If $X$ follows $T-D\{Y\}$ class of distributions, it is easy to see that
(i) $X \stackrel{d}{=} v\left\{\left[F_{Y}(T)\right]^{(-1 / q)}-1\right\}^{(-1 / u)}$
(ii) $Q_{X}(p)=v\left(\left\{F_{Y}\left[Q_{T}(p)\right]\right\}^{(-1 / q)}-1\right)^{(-1 / u)}$
(iii) If $T \stackrel{d}{=} Y$, then $X \stackrel{d}{=} \operatorname{Dagum}(u, q, v)$
(iv) If $Y \stackrel{d}{=} \operatorname{Dagum}(u, q, v)$, then $X^{\stackrel{d}{=}} T$

where $Q_{X}(\cdot)$ is the quantile function of $X, F_{Y}(\cdot)$ is the cdf of $Y$ and $Q_{T}(\cdot)$ is the quantile function of $T$; where $p$ is generated from a standard uniform distribution. Remark 1 (i) is a random variable, while Remark 1(ii) is a quantile function (see [21]).

The cdf in (5) can be used to generate many distributions, who are members of the $T-\mathrm{D}\{\mathrm{Y}\}$ class of distributions. 
2.1. The $T$-\{Lomax $\}$ Family. Let $Y$ be a Lomax random variable with pdf given by

$$
f_{Y}(x)=\frac{\gamma}{\theta}\left(1+\frac{x}{\theta}\right)^{-(\gamma+1)}, \quad x>0, \gamma, \theta>0 .
$$

The cdf and quantile function of Lomax distribution are given by $F_{Y}(x)=1-(1+(x / \theta))^{-\gamma}$ and $Q_{Y}(x)=\theta[(1-$ $\left.p)^{(-1 / \gamma)}-1\right]$, respectively. From equations (5) and (7), the cdf and pdf of the $T-D\{$ Lomax $\}$ distribution are, respectively, given as

$$
\begin{gathered}
f_{X}(x)=F_{T}\left(\theta\left\{1-\left[1+\left(\frac{x}{v}\right)^{-u}\right]^{-q}\right\}^{-(1 / \gamma)}-\theta\right), \\
f_{X}(x) \frac{u q}{x}\left\{\frac{(x / v)^{u q}}{\left[1+(x / v)^{u}\right]^{q+1}}\right\} \\
\times \frac{f_{T}\left(\theta\left\{1-\left[1+(x / v)^{-u}\right]^{-q}\right\}^{-(1 / \gamma)}-\theta\right)}{f_{Y}\left(\theta\left\{1-\left[1+(x / v)^{-u}\right]^{-q}\right\}^{-(1 / \gamma)}-\theta\right)} .
\end{gathered}
$$

The cdf of the $T$-Dagum $\{$ Lomax $\}$ class of distributions, using Lomax quantile function, is defined in (9). This equation (9) is a new way of generalizing Dagum distribution. So, $T$ can be any univariate probability distribution with support $[0, \infty)$.

2.2. Some Properties of $T-D\{L o m a x\}$ Class of Distribution. Some general properties of the $T$-D $\{$ Lomax $\}$ class are discussed in this section.

Lemma 1. Given any random variable $T$ with $p d f f_{T}(x)$, then the random variable $X=v\left\{\left[1-(1+(T / \theta))^{-\gamma}\right]^{-1 / q}-1\right\}^{-1 / u}$ follows T-Dagum\{Lomax\} distribution in (9).

Proof. It is easy to see the result from Remark 1(i). Lemma 1 shows the relationships between $X$ and $T$ random variables. Random variable $X$ can be generated from random variable $T$ using these relationships. If, for instance, random variable $T$ is a known standard random variable, in which quantile function is known, then random variates $X$ can be simulated by first simulating $T$ values.

Lemma 2. The quantile functions for the $T$-D $\{$ Lomax $\}$ distribution is given by

$$
Q_{X}(p)=v\left\{\left[1-\left(1+\left(Q_{T}(p) / \theta\right)\right)^{-\gamma}\right]^{-1 / q}-1\right\}^{-1 / u} .
$$

Proof. It is easy to see the result from Remark 1(ii).

Theorem 1. Shannon's entropy of the T-D $\{$ Lomax $\}$ class of distribution can be expressed as

$$
\begin{aligned}
\eta_{X}= & \eta_{T}+E\left\{\ln \left[f_{Y}(T)\right]\right\}+u q \ln v-\ln u q+E(\ln x) \\
& -u q E(\ln x)+(q+1) E\left\{\ln \left[1+\left(\frac{x}{v}\right)^{u}\right]\right\} .
\end{aligned}
$$

Proof. Since $X^{d}=v\left\{\left[F_{Y}(T)\right]^{-1 / q}-1\right\}^{-1 / u}$, it follows that $T=Q_{Y}\left[F_{R}(x)\right]$. Hence, based on the pdf in equation (3), we can write

$$
f_{X}(x)=f_{R}(x) \frac{f_{T}(t)}{f_{Y}(t)} .
$$

This implies that

$$
\eta_{X}=\eta_{T}+E\left\{\ln \left[f_{Y}(T)\right]\right\}+E\left\{\ln \left[f_{R}(X)\right]\right\} .
$$

For the $T-D\{Y\}$ class of distribution, we have

$$
\ln f_{R}(x)=\ln u q-\ln x+u q \ln x-(q+1) \ln \left[1+\left(\frac{x}{v}\right)^{u}\right] \text {. }
$$

So, the result in Theorem 1 follows from (14) and (15).

\section{Proposed Six-Parameter Exponentiated- Exponential-Dagum $\{$ Lomax $\}$ Distribution}

The six-parameter exponentiated-exponential-Dagum \{Lomax (EEDL) distribution is proposed and we derived some of its characterizations.

3.1. Cumulative Distribution Function (cdf) of EEDL Distribution. Gupta and Kundu [30] defined the pdf of exponentiated exponential distribution as

$$
f_{T}(x)=\alpha \lambda e^{-\lambda x}\left(1-e^{-\lambda x}\right)^{\alpha-1},
$$

where $\lambda$ is the scale parameter and $\alpha$ is the shape parameter, and the cdf is given by

$$
F_{T}(x)=\left(1-e^{-\lambda x}\right)^{\alpha} ; \quad x \geq 0, \alpha, \lambda>0
$$
have

Substituting equations (16) and (17) into equation (9), we

$$
F_{X}(x)=\left\{1-\exp \left[-\lambda \theta\left(\left\{1-\left[\left(1+v^{u} x^{-u}\right)^{-q}\right]\right\}^{-(1 / \gamma)}-1\right)\right]\right\}^{\alpha} .
$$

From (18), it is notable that $\lambda \theta$ is a constant and can be replaced with $\beta$ and $v^{u}$ is also a constant and can be replaced with $\phi$ without any loss of generality so that (18) is reduced to

$$
F_{X}(x)=\left\{1-\exp \left[-\beta\left(\left\{1-\left[\left(1+\phi x^{-u}\right)^{-q}\right]\right\}^{-(1 / \gamma)}-1\right)\right]\right\}^{\alpha},
$$

where $\alpha, \gamma, u$, and $q$ are shape parameters, which define the shape (skewness, kurtosis, and mode) of the distribution, while $\beta$ and $\phi$ are scale parameters, which defines the spread of the distribution. Thus, (19) is now the cdf of the new probability distribution called the exponentiated-exponential-dagum\{lomax (EEDL) distribution.

\subsection{Probability Density Function ( $p d f)$ of EEDL Distribution.} The pdf of the new probability distribution, that is, EEDL distribution can be obtained by differentiating equation (19) 
with respect to $x$ or by substituting equations (8) and (16) directly into equation (10) to have

$$
f_{X}(x)=\frac{\alpha \beta q u \phi}{\gamma} \frac{\exp \left[-\beta\left(\left\{1-\left[\left(1+\phi x^{-u}\right)^{-q}\right]\right\}^{-(1 / \gamma)}-1\right)\right]\left\{1-\exp \left[-\beta\left(\left\{1-\left[\left(1+\phi x^{-u}\right)^{-q}\right]\right\}^{-(1 / \gamma)}-1\right)\right]\right\}^{\alpha-1}}{x^{(u+1)}\left(1+\phi x^{-u}\right)^{q+1}\left\{1-\left[\left(1+\phi x^{-u}\right)^{-q}\right]\right\}^{(1 / \gamma)+1)}}
$$

where $x \geq 0, \alpha, \beta, q, u, \phi, \gamma>0$. Equation (20) is the pdf of the new probability distribution, EEDL. The six-parameter distribution will be a good distribution to model any environmental hazard, survival, and time to failure data parameters.If

$$
\begin{aligned}
w & =\left\{1-\left[\left(1+\phi x^{-u}\right)^{-q}\right]\right\}^{-(1 / \gamma)}-1, \\
w+1 & =\left\{1-\left[\left(1+\phi x^{-u}\right)^{-q}\right]\right\}^{-(1 / \gamma)}, \\
(w+1)^{-\gamma} & =1-\left[\left(1+\phi x^{-u}\right)^{-q}\right], \\
\left(1+\phi x^{-u}\right)^{-q} & =1-(w+1)^{-\gamma}, \\
1+\phi x^{-u} & =\left[1-(w+1)^{-\gamma}\right]^{-(1 / q),}
\end{aligned}
$$

then

$$
\begin{array}{r}
f_{X}(x)=\frac{\alpha \beta q u \phi}{\gamma} \frac{(w+1)^{\gamma+1} e^{-\beta w}\left(1-e^{-\beta w}\right)^{\alpha-1}}{x^{(u+1)}\left(1+\phi x^{-u}\right)^{q+1}}, \\
x, w \geq 0, \alpha, \beta, q, u, \phi, \gamma>0,
\end{array}
$$

and the cdf becomes

$$
F_{X}(x)=\left(1-e^{-\beta w}\right)^{\alpha},
$$

where $w$ is a function of $x$.

3.3. Quantile Function of EEDL Distribution. In probability theory, we can characterize a random variable by its quantile function. It is much important in deriving measures of partition such as the median, quartiles, octiles, deciles, and percentiles.

Lemma 3. The quantile function of the EEDL distribution for $p$ random variable, uniformly distributed on the interval [0, 1], is given by

$$
Q_{X}(p)=\phi^{(1 / u)}\left(\left\{1-\left[1-\frac{1}{\beta} \log \left(1-p^{(1 / \alpha)}\right)\right]^{-\gamma}\right\}^{-(1 / q)}-1\right)^{-(1 / u)}
$$

Proof. Let $p=F(x)=P(X \leq x)$, by making $x$ the subject of the formula in equation (19), and the inverse function of $p$ is given by

$$
x=Q_{X}(p)=\phi^{(1 / u)}\left(\left\{1-\left[1-\frac{1}{\beta} \log \left(1-p^{(1 / \alpha)}\right)\right]^{-\gamma}\right\}^{-(1 / q)}-1\right)^{-(1 / u)}
$$

This quantile function in (25) will be used to generate random variates in the simulation study. The median and 1 st and 3rd quartiles can be obtained by setting $p=0.5,0.25$, and 0.75 , respectively. Other measures of partitions can also be obtained by setting $p$ appropriately.

3.4. Survival Function of EEDL Distribution. If $X$ follows an EEDL distribution and $F_{X}(x)$ be the probability that any given device of interest will survive to a given point in time, $x$, such that $x \in X$, that is, $P(X \leq x)$, then the survival function, $S_{X}(x)$, is a function that gives the probability that such a device will survive beyond $x$.

Suppose that $F_{X}(x)$ is the cdf of EEDL distribution supported on the interval $[0, \infty)$ as proposed in equation (19); then, the survival function of EEDL is given by

$$
\begin{aligned}
S_{X}(x) & =P(X>x) \\
& =1-\left\{1-\exp \left[-\beta\left(\left\{1-\left[\left(1+\phi x^{-u}\right)^{-q}\right]\right\}^{-(1 / \gamma)}-1\right)\right]\right\}^{\alpha},
\end{aligned}
$$

and in terms of $w$, equation (26) becomes

$$
S_{X}(x)=P(X>x)=1-\left(1-e^{-\beta w}\right)^{\alpha} \text {. }
$$

3.5. Hazard Function of EEDL Distribution. Let $X$ be a random variable that follows an EEDL distribution, with pdf and survival function given in (22) and (27), respectively; then, its hazard function is given by

$$
h_{X}(x)=\frac{\alpha \beta q u \phi}{\gamma} \frac{(w+1)^{\gamma+1} e^{-\beta w}\left(1-e^{-\beta w}\right)^{\alpha-1}}{1-\left(1-e^{-\beta w}\right)^{\alpha}},
$$

where $w$ is a function of $x$ as defined in equation (21).

3.6. Cumulative Hazard Function of EEDL Distribution. Let $X$ be a random variable that follows an EEDL distribution, with survival function given in (27), then its cumulative hazard function is given by

$$
H_{X}(x)=-\log \left[1-\left(1-e^{-\beta w}\right)^{\alpha}\right],
$$

where $w$ is a function of $x$ as defined in equation (17).

Figure 1 depicts the pdfs of EEDL distribution for various values of the parameters and depicts that the distribution can be stable (normal), positively skewed, or negatively skewed. Figure 2 depicts that the EEDL distribution can be stable (symmetric), positively or negatively skewed, unimodal, or bimodal. The behaviour of EEDL can 


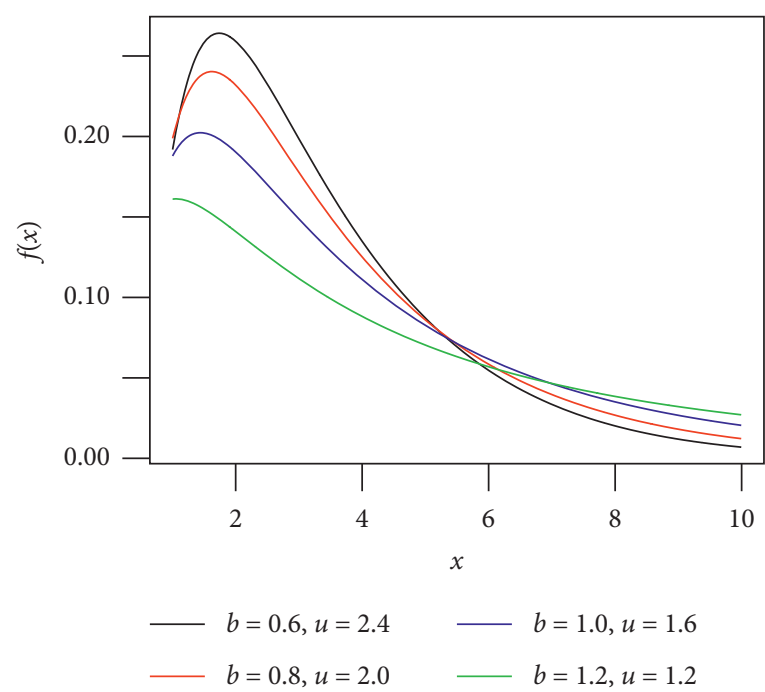

(a)

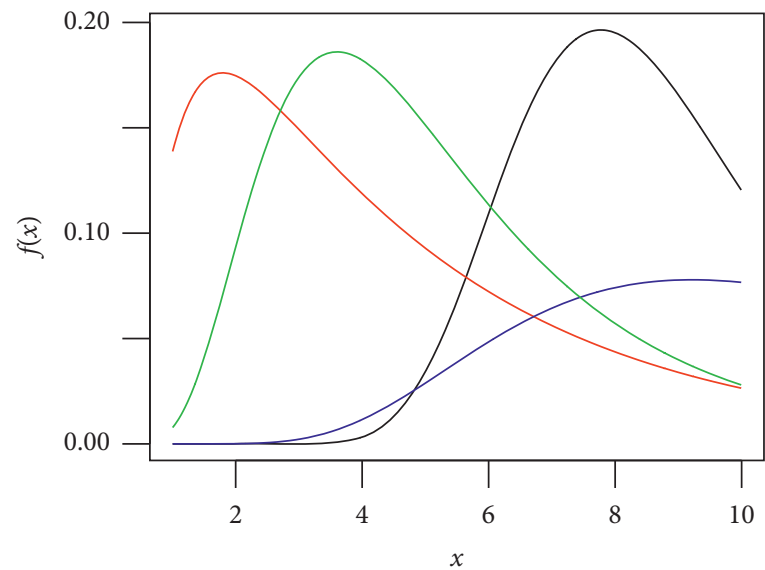

$\begin{aligned}- \text { Alpha } & =15.0, b=0.5, u=2.5 \\ \text { Alpha } & =0.8, b=0.5, u=2.0 \\ \text { Alpha } & =15.0, b=5.0, u=0.8 \\ \text { Alpha } & =5.0, b=5.0, u=1.0\end{aligned}$

(c)

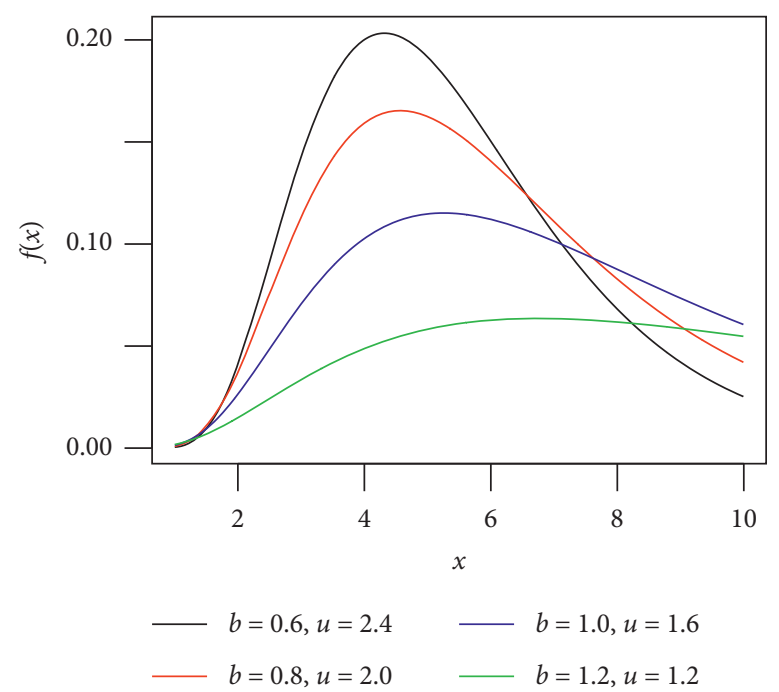

(b)

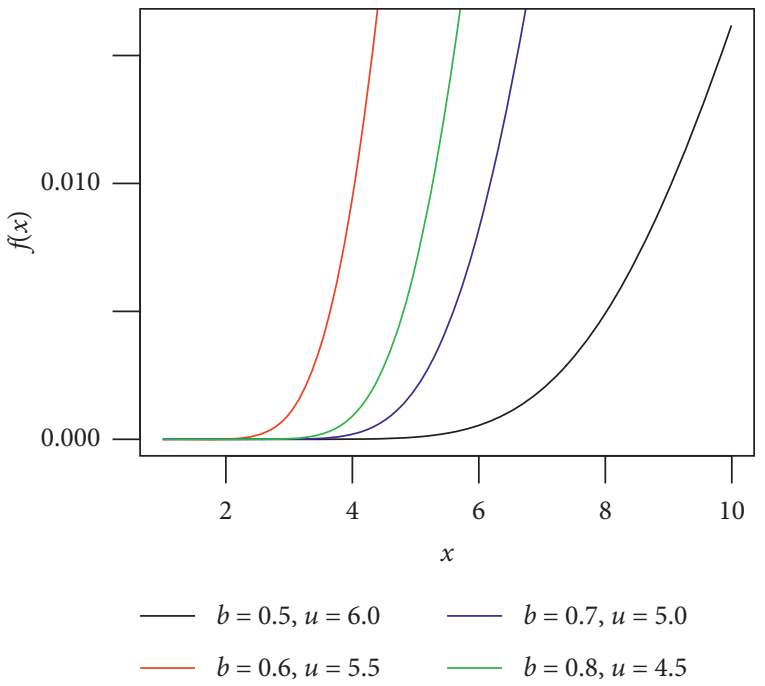

(d)

Figure 1: EEDL probability density function: (a) alpha=0.8, $q=$ phi=gamma=2, (b) alpha=3, $q=$ phi=gamma=2, (c) $q=$ phi= gamma $=2$, and (d) alpha $=8, u=$ phi $=$ gamma $=2$.

help to model any environmental hazard data or any data with high degree of variability. The four shape parameters can capture any features or variation in a dataset.

A distribution can also be characterized by its asymptotes, stochastic ordering, stress, and strength properties.

\subsection{Asymptotes of EEDL Distribution}

\subsubsection{Vertical Asymptotes of EEDL Distribution}

Theorem 2. If $f_{X}(x)$ and $h_{X}(x)$ are the $p d f$ and hazard rate of EEDL distribution, then line $x=(-\phi)^{(1 / u)}$, if it exist, is a vertical asymptote of the graph of the functions $f_{X}(x)$ and $h_{X}(x)$ if the following statements hold:

$$
\lim _{x \longrightarrow(-\phi)^{1 / u}} f_{X}(x)=\infty .
$$

The horizontal asymptote for $h_{X}(x)$ is given by

$$
\lim _{x \longrightarrow(-\phi)^{(1 / u)}} h_{X}(x)=\infty .
$$

Proof. The denominator of the pdf of EEDL distribution in (22) is equated to zero to have

$$
x^{(u+1)}\left(1+\phi x^{-u}\right)^{q+1}=0,
$$

and to solve for $x$, we have 


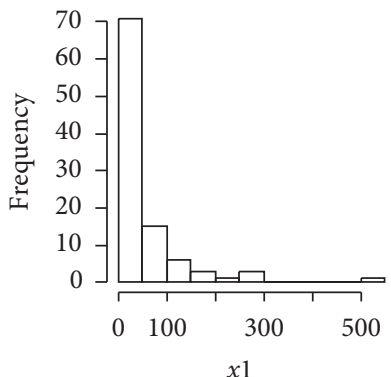

(a)

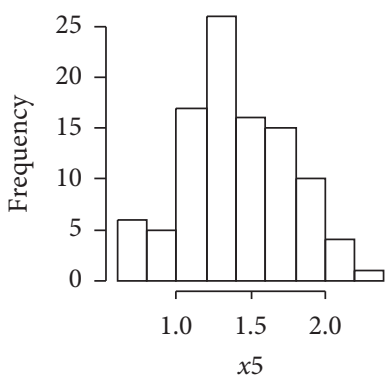

(e)

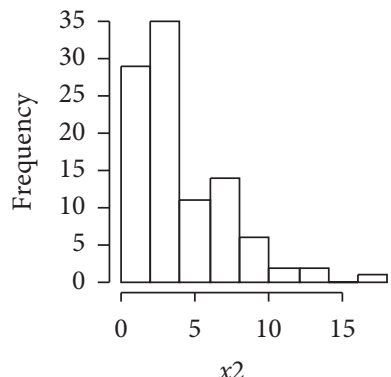

(b)

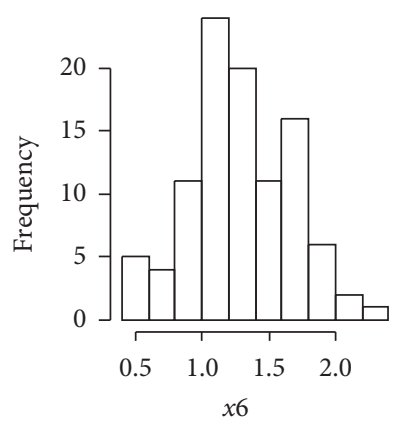

(f)

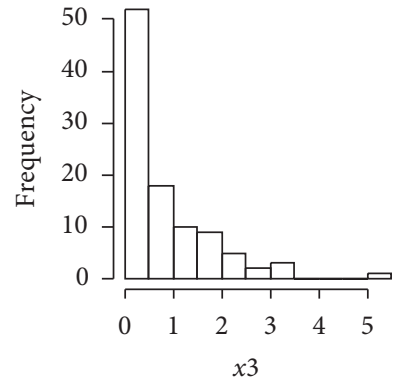

(c)

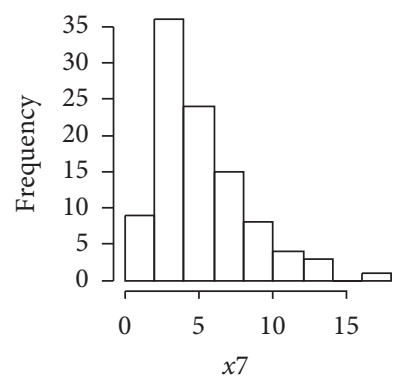

(g)

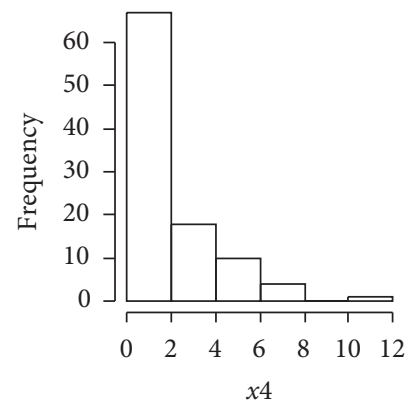

(d)

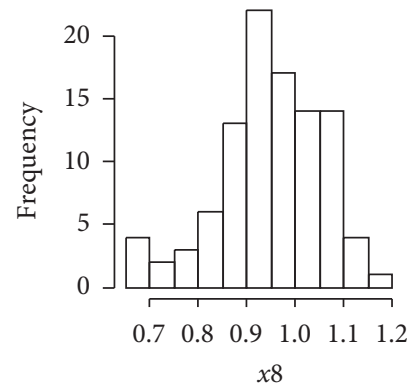

(h)

FIGURE 2: Histogram of EEDL distribution with various parameter values. (a) $\alpha=1.5, \beta=2.5, \gamma=1.5, \phi=3.5, u=0.5$, and $q=1$, (b) $\beta=4.5$, $\gamma=1.5, \phi=2.5, u=0.9$, and $q=2.6$, (c) $\beta=4.5, \gamma=1.5, \phi=2.5$, and $u=0.9$, (d) $\beta=0.5, \gamma=0.5, \phi=0.8$, and $u=1.6,(\mathrm{e}) \phi=2.5, u=3.5$, and $q=1.6$, (f) $\gamma=1.5, \phi=2.5$, and $u=4.2$, (g) $\alpha=5.5$ and $\gamma=1.5$, and (h) $u=10.2$.

$$
\begin{aligned}
\left(1+\phi x^{-u}\right)^{q+1} & =0, \\
\phi x^{-u} & =-1, \\
x & =(-\phi)^{(1 / u)} .
\end{aligned}
$$

Also, the denominator of the hazard rate of EEDL distribution in (28) is equated to zero to have

$$
\begin{aligned}
\gamma\left[1-\left(1-e^{-\beta w}\right)^{\alpha}\right] & =0, \\
\left(1-e^{-\beta w}\right)^{\alpha} & =0, \\
e^{-\beta w} & =1, \\
-\beta w & =\log (1), \\
w & =0,
\end{aligned}
$$

but

$$
\begin{aligned}
w & =\left\{1-\left[\left(1+\phi x^{-u}\right)^{-q}\right]\right\}^{-(1 / \gamma)}-1, \\
\left\{1-\left[\left(1+\phi x^{-u}\right)^{-q}\right]\right\}^{-(1 / \gamma)}-1 & =0, \\
1+\phi x^{-u} & =0, \\
\phi x^{-u} & =-1, \\
x & =(-\phi)^{(1 / u)} .
\end{aligned}
$$

The proof is complete. Note that if $u$ is an even real number, then $x$ is a complex number. Also, if $u$ is an odd real number, then $x$ will be negative because $\phi>0$. Take note that the limit is one-sided, since $x \geq 0$. This implies that the vertical asymptote of EEDL distribution does not exist for all real values of $x \geq 0$.

3.7.2. Horizontal Asymptotes of EEDL Distribution. Let $f_{X}(x)$ and $h_{X}(x)$ be the pdf and hazard functions of EEDL distribution.

The horizontal asymptotes are horizontal lines that the function approaches as $x \longrightarrow \infty$.

The horizontal asymptote for $f_{X}(x)$ is given by

$$
\lim _{x \longrightarrow \infty} f_{X}(x)=0 \text {. }
$$

The horizontal asymptote for $h_{X}(x)$ is given by

$$
\lim _{x \rightarrow \infty} h_{X}(x)=0 \text {. }
$$

Take note that the limit is one-sided, since $x \geq 0$.

\subsection{Stochastic Ordering of EEDL Distribution}

\subsubsection{General Order Statistics of EEDL Distribution}

Theorem 3. The pdf of the general order statistics of EEDL distribution $f_{X_{(j)}}(x)$ exists and it is given by

$$
\begin{aligned}
f_{X_{(j)}}(x)= & \frac{n ! \alpha \beta q u \phi}{\gamma(j-1) !(n-j) !} \\
& \cdot \frac{(w+1)^{\gamma+1} e^{-\beta w}\left(1-e^{-\beta w}\right)^{\alpha j-1}\left[1-\left(1-e^{-\beta w}\right)^{\alpha}\right]^{n-j}}{x^{(u+1)}\left(1+\phi x^{-u}\right)^{q+1}} .
\end{aligned}
$$


Proof. Let $X_{(1)}, X_{(2)}, \ldots, X_{(n)}$ denote the order statistics of a random sample that follows EEDL distribution, from a Substitute (22) and (23) into (39) to have (40): continuous population with $\operatorname{cdf} F_{X}(x)$ and pdf $f_{X}(x)$. Then, the pdf $X_{(j)}$ is

$$
f_{X_{(j)}}(x)=\frac{n !}{(j-1) !(n-j) !} f_{X}(x)\left[F_{X}(x)\right]^{j-1}\left[1-F_{X}(x)\right]^{n-j} .
$$

$$
\begin{aligned}
& f_{X_{(j)}}(x)=\frac{n !}{(j-1) !(n-j) !}\left(\frac{\alpha \beta q u \phi}{\gamma} \frac{(w+1)^{\gamma+1} e^{-\beta w}\left(1-e^{-\beta w}\right)^{\alpha-1}}{x^{(u+1)}\left(1+\phi x^{-u}\right)^{q+1}}\right)\left[\left(1-e^{-\beta w}\right)^{\alpha}\right]^{j-1}\left[1-\left(1-e^{-\beta w}\right)^{\alpha}\right]^{n-j} \\
& f_{X_{(j)}}(x)=\frac{n ! \alpha \beta q u \phi}{\gamma(j-1) !(n-j) !} \frac{(w+1)^{\gamma+1} e^{-\beta w}\left(1-e^{-\beta w}\right)^{\alpha j-1}\left[1-\left(1-e^{-\beta w}\right)^{\alpha}\right]^{n-j}}{x^{(u+1)}\left(1+\phi x^{-u}\right)^{q+1}}
\end{aligned}
$$

Thus, equation (41) completes the proof.

Let $X$ and $Y$ be two random variables that follow the EEDL distribution. $X$ is less than $Y$ if $P(X>x) \leq$ $P(Y>y), \forall x \in(-\infty, \infty)$, where $P($.$) denotes the proba-$ bility of an event.

Theorem 4. Let $X_{1}$ and $X_{2}$ be two random variables that follow the EEDL distribution. If $X_{1} \leq{ }_{s t} X_{2}$ and $E\left(X_{1}\right)=E\left(X_{2}\right)$, then $X_{1} \stackrel{d}{=} X_{2} \quad\left(X_{1}\right.$ is equal to $X_{2}$ in distribution).

Proof. From the pdf of the general order statistic of the EEDL distribution given in (41), set $j=1$ to arrive at the $1^{\text {st }}$ order statistic given by

$$
f_{X_{(1)}}(x)=\frac{n \alpha \beta q u \phi}{\gamma} \frac{(w+1)^{\gamma+1} e^{-\beta w}\left(1-e^{-\beta w}\right)^{\alpha-1}\left[1-\left(1-e^{-\beta w}\right)^{\alpha}\right]^{n-1}}{x^{(u+1)}\left(1+\phi x^{-u}\right)^{q+1}} .
$$

Also, from (41), set $j=2$ to arrive at the $2^{\text {nd }}$ order statistic given by

$$
\begin{aligned}
f_{X_{(2)}}(x)= & \frac{n(n-1) \alpha \beta q u \phi}{\gamma} \\
& \cdot \frac{(w+1)^{\gamma+1} e^{-\beta w}\left(1-e^{-\beta w}\right)^{2 \alpha-1}\left[1-\left(1-e^{-\beta w}\right)^{\alpha}\right]^{n-2}}{x^{(u+1)}\left(1+\phi x^{-u}\right)^{q+1}} .
\end{aligned}
$$

For $X_{(1)} \leq X_{(2)}$, we can show that $E\left(X_{(1)}\right)=E\left(X_{(2)}\right)$ :

$$
\begin{aligned}
& e^{-\beta w}\left(1-e^{-\beta w}\right)^{\alpha-1}\left[1-\left(1-e^{-\beta w}\right)^{\alpha}\right]^{n-1} \\
& \quad \leq(n-1) e^{-\beta w}\left(1-e^{-\beta w}\right)^{2 \alpha-1}\left[1-\left(1-e^{-\beta w}\right)^{\alpha}\right]^{n-2} .
\end{aligned}
$$

Using series expansion, we have the inequality as

$$
\begin{aligned}
& \sum_{i, k=0}(-1)^{i+k}\left(\begin{array}{c}
n-1 \\
i
\end{array}\right)\left(\begin{array}{c}
\alpha i+\alpha-1 \\
k
\end{array}\right) e^{-\beta w(k+1)} \\
& \leq \sum_{i, k=0}(-1)^{i+k}\left(\begin{array}{c}
n-2 \\
i
\end{array}\right)\left(\begin{array}{c}
\alpha i+2 \alpha-1 \\
k
\end{array}\right) e^{-\beta w(k+1)} .
\end{aligned}
$$

Inequality (45) can be reduced to

$$
\sum_{i, k=0}\left(\begin{array}{c}
n-1 \\
i
\end{array}\right)\left(\begin{array}{c}
\alpha i+\alpha-1 \\
k
\end{array}\right) \leq \sum_{i, k=0}\left(\begin{array}{c}
n-2 \\
i
\end{array}\right)\left(\begin{array}{c}
\alpha i+2 \alpha-1 \\
k
\end{array}\right) .
$$

Take the expectation of both sides to have

$$
\begin{aligned}
& E\left[\sum_{i, k=0}\left(\begin{array}{c}
n-1 \\
i
\end{array}\right)\left(\begin{array}{c}
\alpha i+\alpha-1 \\
k
\end{array}\right)\right] \\
& \quad=E\left[\sum_{i, k=0}\left(\begin{array}{c}
n-2 \\
i
\end{array}\right)\left(\begin{array}{c}
\alpha i+2 \alpha-1 \\
k
\end{array}\right)\right] .
\end{aligned}
$$

Note that the expectation of a constant is a constant. To make equality prevail, we test when $i=k=0$ to arrive at

$$
\begin{aligned}
\frac{(n-1) !(\alpha-1) !}{(n-1) !(\alpha-1) !} & =\frac{(n-2) !(2 \alpha-1) !}{(n-2) !(2 \alpha-1) !} \\
1 & =1 .
\end{aligned}
$$

Thus, the proof is complete. Hence, $X_{1}$ and $X_{2}$ are random samples from EEDL distribution.

\subsection{Stress-Strength Reliability Analysis of EEDL Distribution}

Theorem 5. Let $X$ and $Z$ be two independent random variables that follow the EEDL distribution with pdfs $f_{X}(x)$ and $f_{Z}(z)$, respectively. If $Z$ represents the stress and $X$ represents the strength, then the stress-strength reliability of 
EEDL distribution with $X \sim \operatorname{EEDL}\left(\alpha_{1}, \beta_{1}, \phi, u, q, r\right)$ and $Z \sim \operatorname{EEDL}\left(\alpha_{2}, \beta_{2}, \phi, u, q, r\right)$ does not depend on $\beta_{1}$ or $\beta_{2}$.

Proof. The probability that $X>Z$ is the reliability of the stress-strength of the EEDL distribution, and it is given by

$$
\begin{aligned}
& R=P(Z<X)=\int_{0}^{\infty} \int_{0}^{x} f_{X}(x) f_{Z}(z) \mathrm{d} z \mathrm{~d} x, \\
& R=P(Z<X)=\int_{0}^{\infty} f_{X}(x) F_{Z}(x) \mathrm{d} x
\end{aligned}
$$

where

$$
\begin{aligned}
& F_{Z}(z)=\left(1-e^{-\beta w(z)}\right)^{\alpha_{2}} \\
& f_{X}(x)=\alpha_{1} \beta_{1} e^{-\beta_{1} w(x)}\left(1-e^{-\beta_{1} w(x)}\right)^{\alpha_{1}-1}(1+w(x))^{(\gamma+1)} .
\end{aligned}
$$

Put equations (51) and (52) in (50) to have

$$
\begin{aligned}
R= & \int_{0}^{\infty} \alpha_{1} \beta_{1} e^{-\beta_{1} w(x)}\left(1-e^{-\beta_{1} w(x)}\right)^{\alpha_{1}-1}(1+w(x))^{(\gamma+1)} \\
& \cdot\left(1-e^{-\beta w(x)}\right)^{\alpha_{2}} \mathrm{~d} x .
\end{aligned}
$$

By using linear expansion, we have

$$
\begin{aligned}
R= & \alpha_{1} \beta_{1} \sum_{i, j, k}^{\infty}(-1)^{i+j}\left(\begin{array}{c}
\alpha_{2} \\
i
\end{array}\right)\left(\begin{array}{c}
\alpha_{1}-1 \\
j
\end{array}\right)\left(\begin{array}{c}
\gamma+1 \\
k
\end{array}\right) \\
& \cdot \int_{0}^{\infty}[w(x)]^{k} e^{\left(-\beta_{1}+\beta_{2} i+\beta_{1} j\right)} \mathrm{d} x .
\end{aligned}
$$

The integral in (54) using gamma function becomes

$$
\begin{aligned}
R= & \alpha_{1} \beta_{1} \sum_{i, j, k}^{\infty}(-1)^{i+j}\left(\begin{array}{c}
\alpha_{2} \\
i
\end{array}\right)\left(\begin{array}{c}
\alpha_{1}-1 \\
j
\end{array}\right)\left(\begin{array}{c}
\gamma+1 \\
k
\end{array}\right) \\
& \cdot \frac{\Gamma(k+1)}{\left(-\beta_{1}+\beta_{2} i+\beta_{1} j\right)^{k+1}},
\end{aligned}
$$

where $i \leq \alpha_{2}, j \leq \alpha_{1}-1$, and $k \leq \gamma+1$. Equation (55) is the stress-strength reliability function of the EEDL distribution with $X \sim \operatorname{EEDL}\left(\alpha_{1}, \beta_{1}, \phi, u, q, r\right)$ and $X \sim \operatorname{EEDL}\left(\alpha_{2}, \beta_{2}\right.$, $\phi, u, q, r)$.

However, if $i=j=k=0$, equation (55) is reduced to

$$
R=\alpha_{1} \text {. }
$$

Thus, equation (56) completes the proof. Hence, the stress-strength reliability, $R$, does not depend on $\beta$.

The probability density, cumulative distribution, quantile, survival, hazard, cumulative hazard functions, asymptotes, stochastic ordering, and stress-strength analysis are some of the different ways of characterizing a random variable.

3.10. Related Distributions. Most generalized distributions have relationship with their base distributions by varying one or more of its parameters' value. EEDL is also related with its parent distribution.

\subsubsection{Transformations.}

\section{(1) Exponentiated Exponential Distribution}

Lemma 4. If $X \sim \operatorname{EEDL}(\alpha, \beta, \gamma, u, q, \phi)$, then the random variable $Y=\left\{1-\left[\left(1+\phi X^{-u}\right)^{-q}\right]\right\}^{(1 / \gamma)}-1$ has an exponentiated exponential distribution with parameters $\alpha$ and $\beta$.

Proof. By using the transformation method, the result is shown as follows:

$$
f(y)=\alpha \beta e^{-\beta y}\left(1-e^{-\beta y}\right)^{\alpha-1} .
$$

\section{(2) Ratio of Exponential and Lomax Distributions}

Lemma 5. If $X \sim \operatorname{EEDL}(1, \beta, \gamma, u, q, 1)$, then the $p d f$ of random variable $Z=\left\{1-\left[\left(1+\phi X^{-u}\right)^{-q}\right]\right\}^{(1 / \gamma)}-1$ is the ratio of two pdfs of random variables that have an exponential distribution with parameter $\beta$ and Lomax distribution with parameter $\gamma$, respectively.

Proof. By using the transformation method, the result is shown as follows:

$$
f(z)=\frac{\beta e^{-\beta z}}{\gamma(1+z)^{-\gamma}}
$$

3.11. Moments of EEDL Distribution. The moments of a probability distribution are a very important property in describing the distribution. The mean, variance, standard deviation, measure of skewness and kurtosis, and other parametric measures that describe the distribution can be derived from it.

Theorem 6. Let $X$ follow an EEDL distribution, and the rth moment of $X$ can be expressed in terms of gamma function of $W$ with parameters $k+1$ and $\beta(l+1)$, and it is given by

$$
E\left(X^{r}\right)=\frac{\alpha \beta \Gamma(\alpha) A \phi^{(r / u)}}{\Gamma(r / u)} \int_{0}^{\infty} w^{k} e^{-\beta w(1+l)} \mathrm{d} w,
$$

where

$$
A=\sum_{j=0}^{\infty} \sum_{i=0}^{\infty} \sum_{k=0}^{\gamma+1+(j r / q)} \sum_{l=0}^{\alpha-1} \frac{(-1)^{(r / u-j)} \Gamma(\gamma+(j r / q)+1) \Gamma((j / q)+i) \Gamma((r / u)+j)}{\Gamma(j+1) \Gamma(i+1) \Gamma(j / q) \Gamma(\alpha-l) \Gamma(l+1) \Gamma(\gamma+2-k+(j r / q))} .
$$


Proof. Recall that $w=\left\{1-\left[\left(1+\phi x^{-u}\right)^{-q}\right]\right\}^{-(1 / \gamma)}-1$ and $x=\left((1 / \phi)\left\{\left[1-(w+1)^{-\gamma}\right]^{-(1 / q)}-1\right\}\right)^{-(1 / u)}$ so that the $\mathrm{pdf}$ of EEDL distribution is reduced to

$$
\begin{aligned}
& f_{X}(x)=\alpha \beta \frac{e^{-\beta w}\left(1-e^{-\beta w}\right)^{\alpha-1}}{(1+w)^{-(\gamma+1)}}, \\
& f_{X}(x)=\alpha \beta e^{-\beta w}\left(1-e^{-\beta w}\right)^{\alpha-1}(1+w)^{(\gamma+1)} .
\end{aligned}
$$

See the complete proof of equation (62) in Appendix I.

$$
\begin{aligned}
E\left(X^{r}\right) & =\int_{0}^{\infty} x^{r} \alpha \beta e^{-\beta w}\left(1-e^{-\beta w}\right)^{\alpha-1}(1+w)^{(\gamma+1)} \mathrm{d} x, \\
\frac{\delta w}{\delta x} & =\frac{q u \phi x^{-(u+1)\left(1+\phi x^{-u}\right)^{-(q+1)}}}{\gamma(w+1)^{-(\gamma+1)}}, \\
\mathrm{d} x & =\mathrm{d} w \frac{\gamma(w+1)^{-(\gamma+1)} \mathrm{d} x}{q u \phi x^{-(u+1)\left(1+\phi x^{-u}\right)^{-(q+1)}} .}
\end{aligned}
$$

Substitute (64) into (63) to have

$$
\begin{aligned}
& E\left(X^{r}\right)=\alpha \beta \int_{0}^{\infty} \phi^{(r / u)}\left\{\left[1-(1+w)^{-\gamma}\right]^{-(1 / q)}-1\right\}^{-(r / u)} \frac{e^{-\beta w}\left(1-e^{\beta w}\right)^{\alpha-1}}{(1+w)^{-(\gamma+1)}} \mathrm{d} w, \\
& E\left(X^{r}\right)=\alpha \beta \int_{0}^{\infty} \sum_{j=0}^{\infty} \sum_{i=0}^{\infty}(-1)^{(r / u-j)} \frac{\Gamma((j / q)+i) \Gamma((r / u)+j)}{\Gamma(j+1) \Gamma(r / u) \Gamma(i+1) \Gamma((j / q))} \phi^{r / u}(1+w)^{(\gamma+1)}(1+w)^{j \gamma / q} e^{-\beta w}\left(1-e^{\beta w}\right)^{\alpha-1} \mathrm{~d} w, \\
& E\left(X^{r}\right)=\alpha \beta \int_{0}^{\infty} \sum_{j=0}^{\infty} \sum_{i=0}^{\infty} \sum_{k=0}^{\infty} \frac{(-1)^{(r / u-j)} \Gamma(\gamma+(j r / q)+1) \Gamma(j /(q+i)) \Gamma(r /(u+j))}{\Gamma(j+1) \Gamma(r / u) \Gamma(i+1) \Gamma(j / q) \Gamma(k+1) \Gamma(\gamma+2-k+(j r / q))} \phi^{(r / u)} w^{k} e^{-\beta w}\left(1-e^{\beta w}\right)^{\alpha-1} \mathrm{~d} w, \\
& E\left(X^{r}\right)=\frac{\alpha \beta \Gamma(\alpha) A \phi^{(r / u)}}{\Gamma(r / u)} \int_{0}^{\infty} w^{k} e^{-\beta w(1+l)} \mathrm{d} w .
\end{aligned}
$$

Equation (66) completes the proof, where

$$
A=\sum_{j=0}^{\infty} \sum_{i=0}^{\infty} \sum_{k=0}^{\infty} \sum_{l=0}^{\infty} \frac{(-1)^{(r / u-j)} \Gamma(\gamma+(j r / q)+1) \Gamma(j /(q+i)) \Gamma(r / u+j)}{\Gamma(j+1) \Gamma(i+1) \Gamma(j / q) \Gamma(\alpha-l) \Gamma(l+1) \Gamma(\gamma+2-k+(j r / q))} .
$$

Recall gamma function:

$$
\frac{\Gamma(a)}{b^{a}}=\int_{0}^{\infty} x^{a-1} e^{-b x} \mathrm{~d} x .
$$

Thus, the $r$ th moment of the EEDL distribution is given by

$$
E\left(X^{r}\right)=\frac{\alpha \beta \Gamma(\alpha) A \phi^{r / u} \Gamma(k+1)}{\Gamma(r / u)[\beta(l+1)]^{k+1}} .
$$

If the subscripts $i=j=k=l=0$, then equation (69) reduces to

$$
E\left(X^{r}\right)=\frac{\alpha \phi^{r / u}}{\Gamma(\gamma+1)} .
$$

If $r=1$, we have the mean of the EEDL distribution, which does not depend on variables $\beta$ and $q$, and it is given by

$$
E(X)=\frac{\alpha \phi^{(1 / u)}}{\Gamma(\gamma+1)}
$$

and the variance is

$$
V(X)=\frac{\alpha \phi^{(2 / u)}}{\Gamma(\gamma+1)}\left[1-\frac{\alpha}{\Gamma(\gamma+1)}\right] .
$$

3.12. Shannon Entropy of EEDL Distribution. The Shannon entropy of a random variable is a measure of variation of uncertainty. It is defined by [31] as $E\{-\log [f(x)]\}$ for a random variable $X$ with pdf $f(x)$. Let $X$ be a random variable that follows EEDL distribution with pdf $f_{X}(x)$ as given in (62):

$$
f_{X}(x)=\alpha \beta e^{-\beta w}\left(1-e^{-\beta w}\right)^{\alpha-1}(1+w)^{(\gamma+1)} .
$$

The Shannon entropy of EEDL distribution is

$$
\begin{aligned}
& E\left\{-\log \left[\alpha \beta e^{-\beta w}\left(1-e^{-\beta w}\right)^{\alpha-1}(1+w)\right]^{(\gamma+1)}\right\} \\
& \eta_{X}(x)=-\log \alpha-\log \beta+\beta E(w)-(\alpha-1) E \log \left(1-e^{-\beta w}\right)-(\gamma+1) E \log (w+1),
\end{aligned}
$$

where $w$ is a function of $x$. It can be written as $w(x)$ 
3.13. Maximum Likelihood Estimation of EEDL Distribution Parameters. Recall from (20) the pdf of EEDL distribution, and we derived the log likelihood function as

$$
\begin{aligned}
l= & \log L=n \log \Omega+n \log \alpha+n \log \beta \\
& -\beta \sum_{i=1}^{n} w_{i}-u l \sum_{i=1}^{n} \log x_{i}+(\alpha-1) \sum_{i=1}^{n} \log \left(1-e^{-\beta w}\right) .
\end{aligned}
$$

Remember that $w=\left\{1-\left[\left(1+\phi x^{-u}\right)^{-q}\right]\right\}^{-(1 / \gamma)}-1$ and

$$
\Omega=\sum_{i, j, k, l=0}^{\infty} \phi^{l}(-1)^{i+l-j} \frac{\Gamma(i+1) \Gamma(n+l) \Gamma(j / \gamma+k)}{\Gamma(r-i+2) \Gamma(i-j+1) \Gamma(k+1) \Gamma(j+1) \Gamma(j / \gamma) \Gamma(l+1) \Gamma(n)} .
$$

We can differentiate (75) easily with respect to $\alpha$ and $\beta$. Differentiate (75) partially with respect to $\alpha$, and equate the result to zero and solve for $\alpha$ :

$$
\begin{gathered}
\frac{\delta l}{\delta \alpha}=\frac{n}{\alpha}+\sum_{i=1}^{n} \log \left(1-e^{-\beta w_{i}}\right), \\
0=\frac{n}{\alpha}+\sum_{i=1}^{n} \log \left(1-e^{-\beta w_{i}}\right), \\
-\sum_{i=1}^{n} \log \left(1-e^{-\beta w_{i}}\right)=\frac{n}{\alpha}, \\
\widehat{\alpha}=\frac{-n}{\sum_{i=1}^{n} \log \left(1-e^{-\beta w_{i}}\right)} .
\end{gathered}
$$

Differentiate (75) partially with respect to $\beta$, equate the result to zero and solve for $\beta$ :

$$
\begin{aligned}
\frac{\delta l}{\delta \beta} & =\frac{n}{\beta}-\sum_{i=1}^{n} w_{i}+\beta(\alpha-1) \sum_{i=1}^{n} \frac{e^{-\beta x_{i}}}{1-e^{-\beta x_{i}}}, \\
0 & =\frac{n}{\beta}-\sum_{i=1}^{n} w_{i}+\beta(\alpha-1) \sum_{i=1}^{n} \frac{e^{-\beta x_{i}}}{1-e^{-\beta x_{i}}}, \\
\sum_{i=1}^{n} w_{i} & =\frac{n}{\beta}+\beta(\alpha-1) \sum_{i=1}^{n} \frac{e^{-\beta x_{i}}}{1-e^{-\beta x_{i}} .}
\end{aligned}
$$

Divide through by $n$

$$
\bar{w}=\frac{1}{\beta}+\frac{(\alpha-1) \beta}{n} \sum_{i=1}^{n} \frac{e^{-\beta x_{i}}}{\left(1-e^{-\beta x_{i}}\right)}
$$

The estimate of $\beta$ is not in a closed form. From (58), we also differentiate partially with respect to other parameters, equate their results to zero, and solve for each of them. Their solutions are not in closed form as well, so we resolved by using R package "MaxLik."

3.14. Simulation. A simulation study is carried out to investigate the performance of the estimators. The standard error of estimate (SE), average absolute bias (AAB), and root mean square error (RMSE) of the maximum likelihood estimators of the parameters of the EEDL were examined. The simulation study was repeated for $N=1000$ times each with sample sizes $n=20,50,100,250$, and 500 and parameter values: $\alpha=1.5, \beta=0.1, \gamma=2.0, \phi=0.3, q=0.01$, and $u=0.9$.

Table 1 presents the MLE estimates, standard error of estimate, and $\mathrm{AAB}$ and RMSE values of the parameters $\alpha, \beta$, $\gamma, \phi, q$, and $u$ for different sample sizes. The result shows that as the sample size approaches infinity, the AAB and RMSE decrease asymptotically to zero, proving their consistency. It is consistent in the sense that it converges to the true parameter value as the number of observations becomes larger and the error reduces to zero. See Figure 3 for pictorial view.

3.15. Applications. The EEDL distribution is fitted to two real datasets retrieved from [13]. The first application is a data on failure times for 36 appliances subjected to an automatic life test, while the second data is on failure time data on $100 \mathrm{~cm}$ yarn subjected to $2: 3 \%$ strain level. EEDL distribution is compared with that of exponentiated generalized exponential Dagum distribution (EGEDD), the exponentiated Kumaraswamy Dagum (EKD) distribution and the Mc-Dagum (McD) distribution using Log-likelihood, Akaike information criterion (AIC), and Kolmogorov-Smirnov (K-S) statistic criteria.

3.15.1. Application 1: Appliances Data. The appliances data in Table 2 was obtained from $[13,32]$. The dataset consists of failure times for 36 appliances subjected to an automatic life test. The dataset is depicted in Figure 4 and shows that there is a gap in the histogram with positive skewness (2.279) and highly leptokurtic (9.669). Table 3 displays the maximum likelihood estimates of the parameters with their corresponding standard errors in brackets. Table 3 shows all the parameters of the EEDL distribution and other distributions.

Table 4 clearly shows that the EEDL distribution provides a better fit to the appliances data than the other models. Its log-likelihood, AIC and approach zero faster than other distributions. It also has the smallest K-S statistic compared with other distribution in this research. Thus, the EEDL is a better fit to the appliances data. 
TABLE 1: Simulation results of MLE.

\begin{tabular}{|c|c|c|c|c|c|}
\hline Parameter & $n$ & Estimate & SE & $\mathrm{AAB}$ & RMSE \\
\hline \multirow{5}{*}{$\alpha=1.5$} & 20 & 1.5020 & 0.0000549 & 0.001610 & 0.00720 \\
\hline & 50 & 1.5013 & 0.0000204 & 0.000660 & 0.00466 \\
\hline & 100 & 1.5017 & 0.0000132 & 0.000360 & 0.00357 \\
\hline & 250 & 1.5020 & 0.0000032 & 0.000120 & 0.00184 \\
\hline & 500 & 1.5001 & 0.0000001 & 0.000010 & 0.00010 \\
\hline \multirow{5}{*}{$\beta=0.1$} & 20 & 0.0367 & 1.0443120 & 0.222721 & 0.99604 \\
\hline & 50 & 0.1447 & 0.3889741 & 0.087315 & 0.61741 \\
\hline & 100 & 0.1437 & 0.1935787 & 0.043777 & 0.43777 \\
\hline & 250 & 0.1596 & 0.0765768 & 0.017467 & 0.27617 \\
\hline & 500 & 0.4500 & 0.0002455 & 0.000700 & 0.01565 \\
\hline \multirow{5}{*}{$\gamma=2.0$} & 20 & 2.0110 & 0.0142591 & 0.026025 & 0.11639 \\
\hline & 50 & 2.0111 & 0.0053693 & 0.010259 & 0.07254 \\
\hline & 100 & 2.0013 & 0.0026316 & 0.005104 & 0.05104 \\
\hline & 250 & 2.0010 & 0.0010761 & 0.002071 & 0.03274 \\
\hline & 500 & 2.0001 & 0.0005010 & 0.001000 & 0.02236 \\
\hline \multirow{5}{*}{$\phi=0.3$} & 20 & 0.3015 & 0.3286991 & 0.124953 & 0.55881 \\
\hline & 50 & 0.3012 & 0.1237652 & 0.049252 & 0.34827 \\
\hline & 100 & 0.3011 & 0.0626044 & 0.024895 & 0.24895 \\
\hline & 250 & 0.2996 & 0.0246000 & 0.009900 & 0.15653 \\
\hline & 500 & 0.2999 & 0.0001804 & 0.000600 & 0.01342 \\
\hline \multirow{5}{*}{$q=0.01$} & 20 & 0.0002 & 0.0624754 & 0.054476 & 0.24362 \\
\hline & 50 & 0.0154 & 0.0241751 & 0.021768 & 0.15392 \\
\hline & 100 & 0.0130 & 0.0119727 & 0.010887 & 0.10887 \\
\hline & 250 & 0.0116 & 0.0047506 & 0.004350 & 0.06879 \\
\hline & 500 & 0.0101 & 0.0000000 & 0.000000 & 0.00000 \\
\hline \multirow{5}{*}{$u=0.9$} & 20 & 0.9014 & 0.0000152 & 0.000849 & 0.00379 \\
\hline & 50 & 0.9008 & 0.0000052 & 0.000319 & 0.00226 \\
\hline & 100 & 0.9005 & 0.0000021 & 0.000144 & 0.00144 \\
\hline & 250 & 0.9002 & 0.0000012 & 0.000070 & 0.00111 \\
\hline & 500 & 0.9001 & 0.0000010 & 0.000001 & 0.00000 \\
\hline
\end{tabular}

3.15.2. Application 2: Yarn Data. Table 5 represents the data on time to failure of a $100 \mathrm{~cm}$ polyster/viscose yarn subjected to $2: 3 \%$ strain level in textile experiment in order to assess the tensile fatigue characteristics of the yarn. The dataset can be found in $[13,33,34]$. The skewness and kurtosis of the data are 1.336119 and 5.802452, respectively. The data is positively skewed and very peaked, as depicted in Figure 5.

The maximum likelihood estimates of the parameters of the fitted models with their corresponding standard errors in brackets are given in Table 6. All the parameters of the EEDL are significant at the $5 \%$ significance level. The EEDL provides a better fit to the yarn data than the EGEDD, EKD, and $\mathrm{McD}$ distributions, as shown in Table 7.

Table 7 shows that EEDL log-likelihood and its AIC approaches zero than that of others and has the smallest K-S statistic compared to the other models. Thus, the EEDL is a better fit.

The kernel density graphs of the data and the EEDL distribution are super-imposed on the histograms on Figures 4 and 5. This shows that the newly proposed EEDL distribution fits the two data.

Figures 6 to 9 show that EEDL distribution fit the two data well. The EEDL distribution fits the yarn data more than the appliances data. The QQ plots in Figures 10 and 11 shows that most of the data points, especially the ones at the middle, fall on the theoretical line, but the few ones outside the unit circle ( -1 and 1$)$ of the theoretical quantiles fall outside the line. This means that if the edges of the data are trimmed, the EEDL distribution would be a perfect fit for the data. However, Tables 4 and 7 shows a confirmatory test that the EEDL distribution is a good fit to both the appliances and yarn data via the K-S statistic and $p$ value.

\section{Concluding Remarks}

This research proposed a new univariate continuous probability distribution called exponentiated-exponentialDagum \{Lomax distribution, EEDL, which is a member of the $T$-Dagum $\{$ lomax $\}$ family and presented results on its statistical properties, such as the cumulative distribution function, density function, the quantile function, survival function, hazard function, cumulative hazard function, asymptotes, stochastic ordering, stress-strength analysis, moments, and Shannon entropy. The maximum likelihood estimation of the parameters of the model was derived. The newly proposed EEDL distribution was applied to two datasets that are positively skewed and very peaked and the results of its performance were compared favourably with EGEDD, EKD, and McD. Further research can be carried out on submodels of EEDL.

See Table 8 for seven of its submodels with fewer parameters. These submodels will be investigated in 

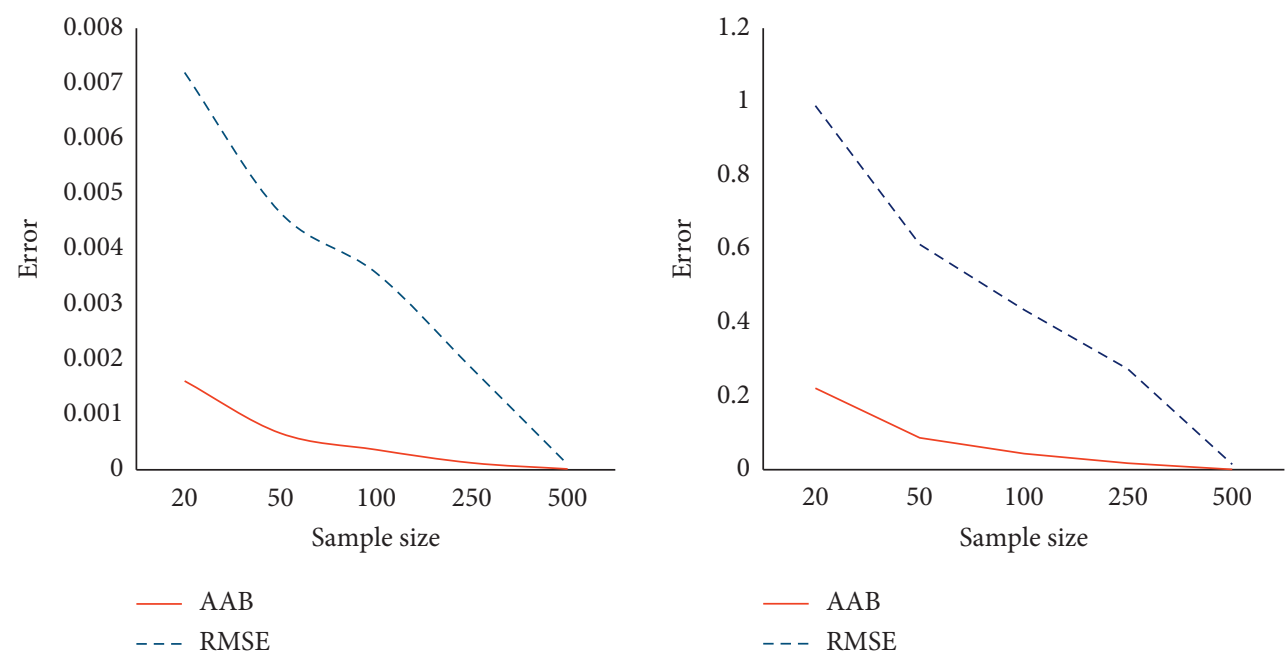

(a)
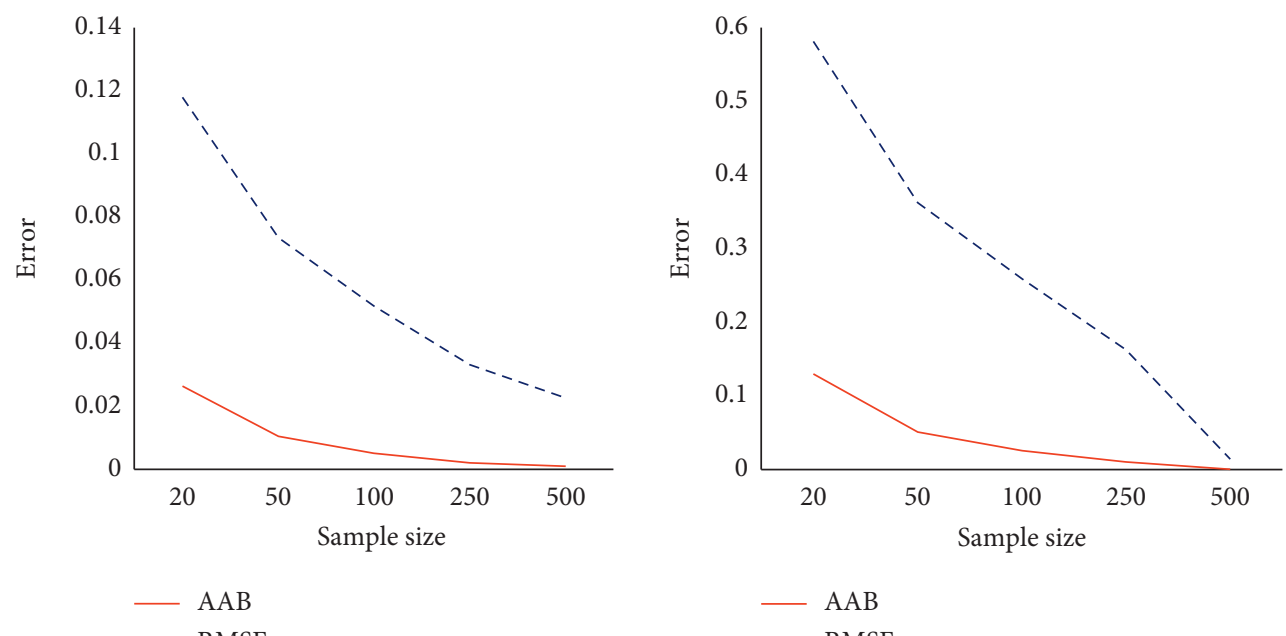

- - RMSE

(c)

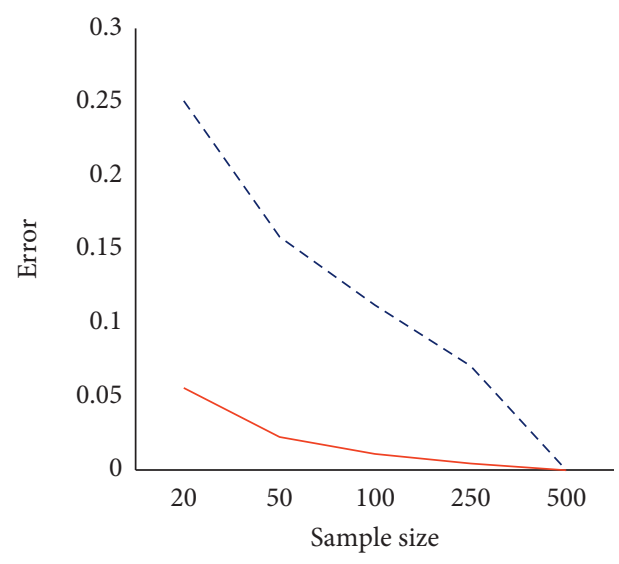

(d)

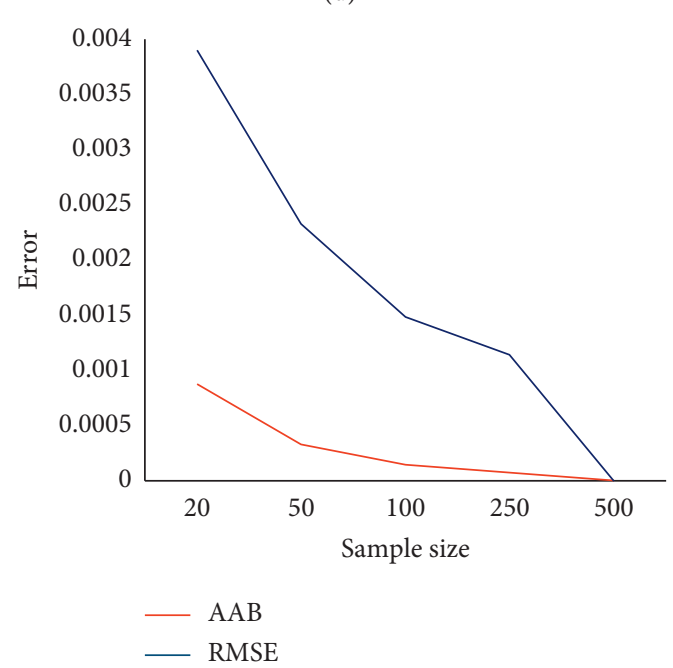

(e)

(f)

Figure 3: (a) $\alpha=1.5$. (b) $\beta=0.1$. (c) $\gamma=2.0$. (d) $\phi=0.3$. (e) $q=0.01$. (f) $u=0.9$. 
TABLE 2: Failure times for 36 appliances subjected to an automatic life test.

\begin{tabular}{lccccccccccc}
\hline 11 & 35 & 49 & 170 & 1990 & 2831 & 2223 & 3034 & 2327 & 3059 & 2400 & 3112 \\
329 & 2451 & 3214 & 381 & 2471 & 3478 & 708 & 2551 & 3504 & 958 & 2565 & 4329 \\
1062 & 2568 & 6367 & 1167 & 2694 & 6976 & 1594 & 2702 & 7846 & 1925 & 2761 & 13403 \\
\hline
\end{tabular}

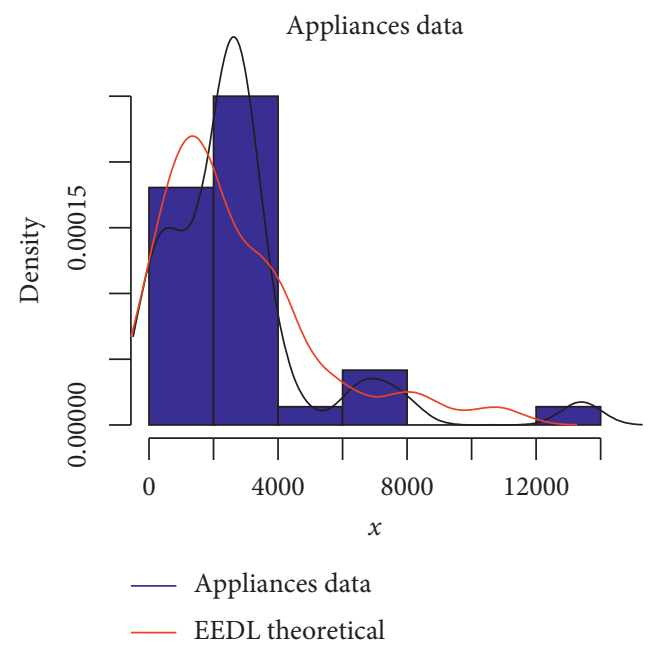

FIgURE 4: Failure time on appliances.

TABLE 3: MLE of parameters and standard errors for appliances data.

\begin{tabular}{|c|c|c|c|c|c|c|}
\hline \multirow[t]{2}{*}{ Model } & \multicolumn{6}{|c|}{ Parameter estimates } \\
\hline & $\hat{\alpha}$ & $\hat{\beta}$ & $\hat{\gamma}$ & $\widehat{\phi}$ & $\hat{q}$ & $\widehat{u}$ \\
\hline EEDL & $\begin{array}{c}0.491 \\
(0.0822) \\
\end{array}$ & $\begin{array}{c}0.011 \\
(0.0001) \\
\end{array}$ & $\begin{array}{c}0.260 \\
(0.0189) \\
\end{array}$ & $\begin{array}{c}1.055 \\
(0.0729) \\
\end{array}$ & $\begin{array}{c}1.041 \\
(0.0666) \\
\end{array}$ & $\begin{array}{c}0.163 \\
(0.0163) \\
\end{array}$ \\
\hline & $\widehat{\alpha}$ & $\hat{\lambda}$ & $\widehat{\beta}$ & $\hat{\theta}$ & $\hat{c}$ & $\widehat{d}$ \\
\hline EGEDD & $\begin{array}{c}0.001 \\
(0.0001)\end{array}$ & $\begin{array}{l}27.198 \\
(0.001) \\
\end{array}$ & $\begin{array}{c}4.560 \\
(0.847) \\
\end{array}$ & $\begin{array}{c}2.838 \\
(0.123) \\
\end{array}$ & $\begin{array}{l}20.866 \\
(0.010) \\
\end{array}$ & $\begin{array}{c}0.070 \\
(0.003) \\
\end{array}$ \\
\hline & $\widehat{\alpha}$ & $\hat{\lambda}$ & $\widehat{\delta}$ & $\widehat{\phi}$ & $\widehat{\theta}$ & \\
\hline EKD & $\begin{array}{c}5.562 \\
(1.517) \\
\end{array}$ & $\begin{array}{l}12.683 \\
(2.158) \\
\end{array}$ & $\begin{array}{c}3.716 \\
(0.755) \\
\end{array}$ & $\begin{array}{c}0.128 \\
(0.029) \\
\end{array}$ & $\begin{array}{l}11.609 \\
(3.922) \\
\end{array}$ & \\
\hline & $\hat{\lambda}$ & $\widehat{\delta}$ & $\widehat{\beta}$ & $\hat{a}$ & $\widehat{b}$ & $\widehat{c}$ \\
\hline $\mathrm{McD}$ & $\begin{array}{c}1.427 \\
(0.092)\end{array}$ & $\begin{array}{c}3.455 \\
(0.212)\end{array}$ & $\begin{array}{c}1.275 \\
(6.875)\end{array}$ & $\begin{array}{c}10.505 \\
(56.906)\end{array}$ & $\begin{array}{c}0.064 \\
(0.012)\end{array}$ & $\begin{array}{l}500.556 \\
(6.976)\end{array}$ \\
\hline
\end{tabular}

TABLE 4: Log-likelihood, information, and goodness-of-fit criteria for appliances data.

\begin{tabular}{lcccc}
\hline Model & LogLik & AIC & K-S stat & KS $p$ value \\
\hline EEDL & $-\mathbf{3 2 0 . 7 5 6}$ & $\mathbf{6 5 3 . 5 1 2}$ & $\mathbf{0 . 2 2 2}$ & $\mathbf{0 . 3 4 0}$ \\
EGEDD & -328.870 & 669.740 & 0.253 & 0.124 \\
EKD & -341.650 & 693.295 & 0.269 & 0.178 \\
McD & -356.480 & 724.955 & 0.347 & 0.128 \\
\hline
\end{tabular}

TABLE 5: Failure time data on $100 \mathrm{~cm}$ yarn subjected to $2: 3 \%$ strain level.

\begin{tabular}{|c|c|c|c|c|c|c|c|c|c|}
\hline 86 & 146 & 251 & 653 & 98 & 249 & 400 & 292 & 131 & 169 \\
\hline 175 & 176 & 76 & 264 & 15 & 364 & 195 & 262 & 88 & 264 \\
\hline 157 & 220 & 42 & 321 & 180 & 198 & 38 & 20 & 61 & 121 \\
\hline 282 & 224 & 149 & 180 & 325 & 250 & 196 & 90 & 229 & 166 \\
\hline 38 & 337 & 65 & 151 & 341 & 40 & 40 & 135 & 597 & 246 \\
\hline 211 & 180 & 93 & 315 & 353 & 571 & 124 & 279 & 81 & 286 \\
\hline 497 & 182 & 423 & 185 & 229 & 400 & 338 & 290 & 398 & 71 \\
\hline 246 & 185 & 188 & 568 & 55 & 55 & 61 & 244 & 20 & 289 \\
\hline 393 & 396 & 203 & 829 & 239 & 236 & 286 & 194 & 277 & 143 \\
\hline 198 & 264 & 105 & 203 & 124 & 137 & 135 & 350 & 193 & 188 \\
\hline
\end{tabular}




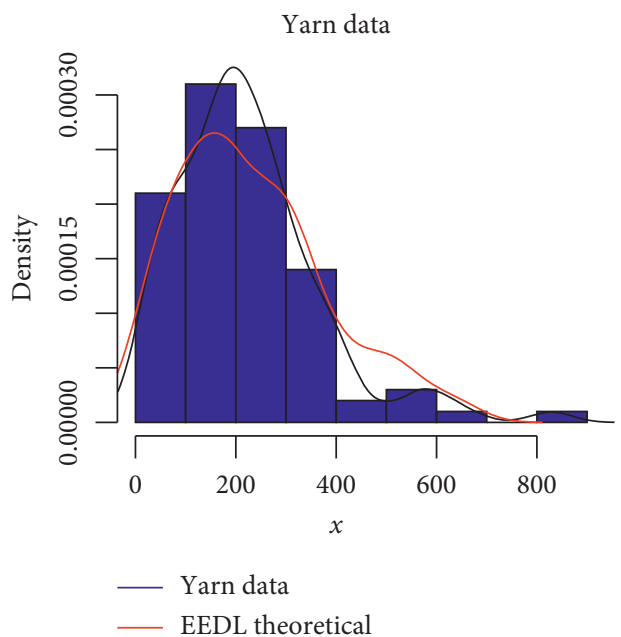

FIGURE 5: Failure time on yarn.

TABLE 6: Maximum likelihood estimates of parameters and standard errors for yarn data.

\begin{tabular}{|c|c|c|c|c|c|c|}
\hline \multirow{3}{*}{$\begin{array}{l}\text { Model } \\
\text { EEDL }\end{array}$} & \multicolumn{6}{|c|}{ Parameter estimates } \\
\hline & $\widehat{\alpha}$ & $\widehat{\beta}$ & $\hat{\gamma}$ & $\widehat{\phi}$ & $\hat{q}$ & $\widehat{u}$ \\
\hline & $\begin{array}{c}1.0921 \\
(0.2133)\end{array}$ & $\begin{array}{c}0.0004 \\
(0.0004) \\
\end{array}$ & $\begin{array}{c}1.5196 \\
(0.1457) \\
\end{array}$ & $\begin{array}{c}3.4906 \\
(0.0005) \\
\end{array}$ & $\begin{array}{c}0.7205 \\
(0.0924) \\
\end{array}$ & $\begin{array}{c}2.3297 \\
(0.0228)\end{array}$ \\
\hline \multirow[b]{2}{*}{ EGEDD } & $\hat{\alpha}$ & $\hat{\lambda}$ & $\widehat{\beta}$ & $\hat{\theta}$ & $\widehat{c}$ & $\bar{d}$ \\
\hline & $\begin{array}{c}0.026 \\
(0.007)\end{array}$ & $\begin{array}{l}75.310 \\
(0.007)\end{array}$ & $\begin{array}{c}0.017 \\
(0.005)\end{array}$ & $\begin{array}{l}3.513 \\
(0.631)\end{array}$ & $\begin{array}{l}45.692 \\
(0.036)\end{array}$ & $\begin{array}{c}0.090 \\
(0.011)\end{array}$ \\
\hline \multirow[b]{2}{*}{ EKD } & $\bar{\alpha}$ & $\bar{\lambda}$ & $\widehat{\delta}$ & $\hat{\phi}$ & $\hat{\theta}$ & \\
\hline & $\begin{array}{l}46.109 \\
(1.295)\end{array}$ & $\begin{array}{l}39.413 \\
(5.006)\end{array}$ & $\begin{array}{l}5.188 \\
(0.961)\end{array}$ & $\begin{array}{c}0.203 \\
(0.040)\end{array}$ & $\begin{array}{c}31.169 \\
(11.023)\end{array}$ & \\
\hline \multirow[b]{2}{*}{$\mathrm{McD}$} & $\hat{\lambda}$ & $\widehat{\delta}$ & $\bar{\beta}$ & $\hat{a}$ & $\widehat{b}$ & $\bar{c}$ \\
\hline & $\begin{array}{c}0.027 \\
(0.0848)\end{array}$ & $\begin{array}{c}0.600 \\
(0.09647)\end{array}$ & $\begin{array}{c}98.780 \\
(0.00002) \\
\end{array}$ & $\begin{array}{c}0.333 \\
(0.1504) \\
\end{array}$ & $\begin{array}{c}25.042 \\
(0.0004507) \\
\end{array}$ & $\begin{array}{c}46.276 \\
(0.00004654) \\
\end{array}$ \\
\hline
\end{tabular}

TABLe 7: Log-likelihood, information, and goodness-of-fit criteria for yarn data.

\begin{tabular}{lcccc}
\hline Model & LogLik & AIC & K-S stat & KS $p$ value \\
\hline EEDL & $\mathbf{- 6 2 5 . 4 5 9}$ & $\mathbf{1 9 9 . 7 1 9}$ & $\mathbf{0 . 1 1 0}$ & $\mathbf{0 . 5 8 1}$ \\
EGEDD & -628.170 & 1268.336 & 0.249 & 0.124 \\
EKD & -653.960 & 1317.913 & 0.985 & 0.178 \\
McD & -628.200 & 1268.399 & 0.285 & 0.128 \\
\hline
\end{tabular}

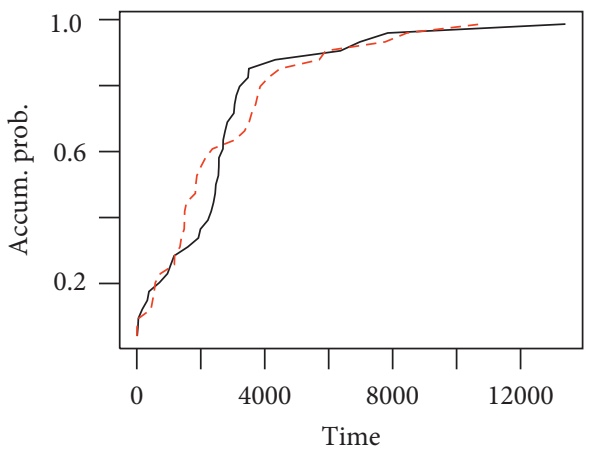

- Appliances data

- - - EEDL

FIgURE 6: TTT plot of appliances.

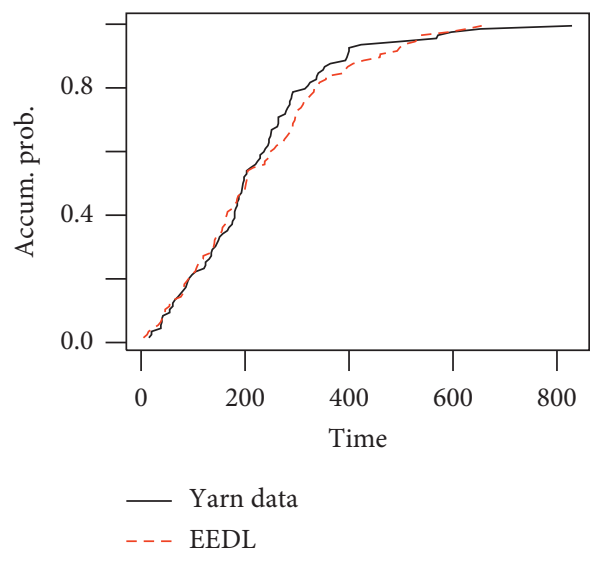

Figure 7: TTT plot of yarn. 


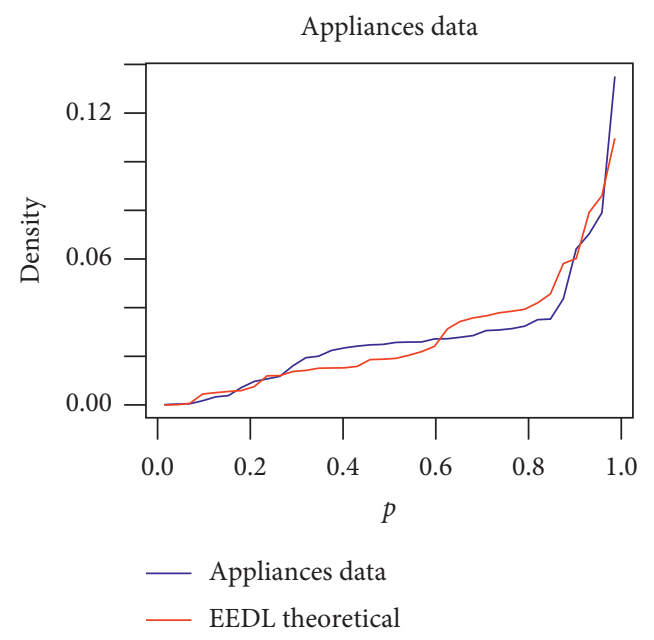

Figure 8: PP plot of appliances.

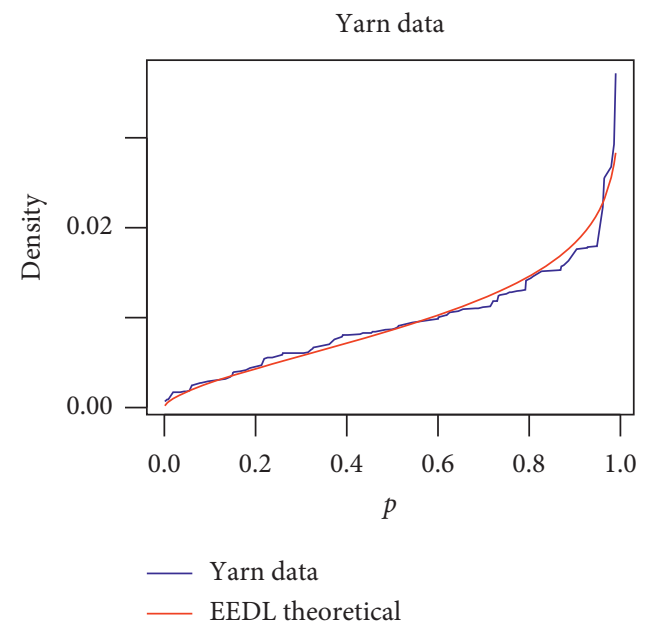

Figure 9: PP plot of yarn.

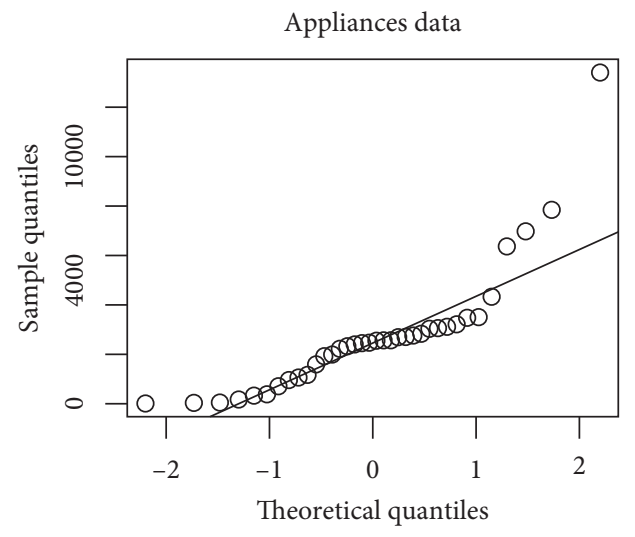

FIgURE 10: QQ plot of appliances.

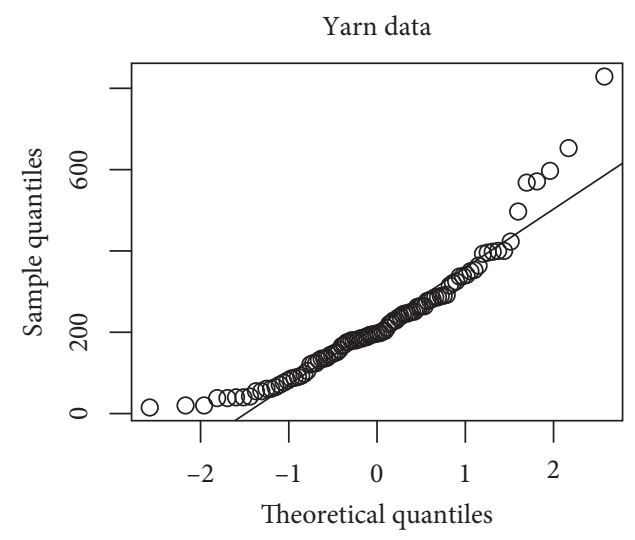

FIgURE 11: QQ plot for EEDL distribution on yarn data. 
TABLE 8: Submodels of EEDL distribution.

\begin{tabular}{|c|c|c|}
\hline SM & Density function & $\mathrm{Np}$ \\
\hline 1 & $f_{X}(x)=(\beta q u \phi / \gamma)\left(\exp \left[-\beta\left(\left\{1-\left[\left(1+\phi x^{-u}\right)^{-q}\right]\right\}^{-(1 / \gamma)}-1\right)\right]\right) /\left(x^{(u+1)}\left(1+\phi x^{-u}\right)^{q+1}\left\{1-\left[\left(1+\phi x^{-u}\right)^{-q}\right]\right\}^{((1 / \gamma)+1)}\right), \alpha=1$ & 5 \\
\hline 2 & $f_{X}(x)=(\beta u \phi) /(\gamma)\left(\exp \left[-\beta\left(\left\{1-\left[\left(1+\phi x^{-u}\right)^{-q}\right]\right\}^{-(1 / \gamma)}-1\right)\right]\right) / x^{(u+1)}\left(1+\phi x^{-u}\right)^{2}\left\{1-\left[\left(1+\phi x^{-u}\right)^{-1}\right]\right\}^{((1 / \gamma)+1)}, \alpha=q=1$ & 4 \\
\hline 3 & $f_{X}(x)=\beta q u \phi\left(\exp \left[-\beta\left(\left\{1-\left[\left(1+\phi x^{-u}\right)^{-q}\right]\right\}^{-1}-1\right)\right]\right) / x^{(u+1)}\left(1+\phi x^{-u}\right)^{q+1}\left\{1-\left[\left(1+\phi x^{-u}\right)^{-q}\right]\right\}^{2}, \alpha=\gamma=1$ & 4 \\
\hline 4 & $f_{X}(x)=q u \phi\left(\exp \left[-\left(\left\{1-\left[\left(1+\phi x^{-u}\right)^{-q}\right]\right\}^{-1}-1\right)\right]\right) / x^{(u+1)}\left(1+\phi x^{-u}\right)^{q+1}\left\{1-\left[\left(1+\phi x^{-u}\right)^{-q}\right]\right\}^{2}, \alpha=\gamma=\beta=1$ & 3 \\
\hline 5 & $f_{X}(x)=(u \phi / \gamma)\left(\exp \left[-\left(\left\{1-\left[\left(1+\phi x^{-u}\right)^{-1}\right]\right\}^{-(1 / \gamma)}-1\right)\right] / x^{(u+1)}\left(1+\phi x^{-u}\right)^{2}\left\{1-\left[\left(1+\phi x^{-u}\right)^{-1}\right]\right\}^{((1 / \gamma)+1)}\right), \alpha=q=\beta=1$ & 3 \\
\hline 6 & $\begin{array}{c}f_{X}(x)=(\alpha / \gamma)\left(\exp _{\alpha-1}\left[-\left(\left\{1-\left[\left(1+\phi x^{-1}\right)^{-1}\right]\right\}^{-(1 / \gamma)}-1\right)\right]\left\{1-\exp \left[-\left(\left\{1-\left[\left(1+\phi x^{-1}\right)^{-1}\right]\right\}^{-(1 / \gamma)}-1\right)\right]\right\}\right. \\
\left.\left.x^{-u}\right)^{2}\left\{1-\left[\left(1+\phi x^{-1}\right)^{-1}\right]\right\}^{((1 / \gamma)+1)}\right), \phi=\beta=u=q=1\end{array}$ & 2 \\
\hline 7 & $f_{X}(x)=u\left(\exp \left[-\left(\left\{1-\left[\left(1+\phi x^{-u}\right)^{-1}\right]\right\}^{-(1 / \gamma)}-1\right)\right] / x^{(u+1)}\left(1+x^{-u}\right)^{2}\left\{1-\left[\left(1+x^{-u}\right)^{-1}\right]\right\}^{2}\right), \alpha=\gamma=\phi=\beta=q=1$ & 1 \\
\hline
\end{tabular}

SM denotes submodel and Np denotes number of parameters. Model 1 is exponential-Dagum\{Lomax $\}$ distribution (EDD) with parameters $\beta, \gamma, \phi, q$, and $u$; model 2 is a four-parameter EDD with parameters $\beta, \gamma, \phi$, and $u$; model 3 is a four-parameter EDD with parameters $\beta$, $\phi$, $u$, and $q$; model 4 is a threeparameter EDD with parameters $\phi, q$, and $u$; model 5 is a three-parameter EDD with parameters $\gamma$, $\phi$, and $u$; model 6 is a two-parameter EEDL distribution with parameters $\alpha$ and $\gamma$; model 7 is a one-parameter EEDL distribution with parameter $u$.

subsequent work. Exponential-DagumLomax distribution is one of its submodels. The more significant a parameter of a distribution is, the more likely its fit is better to some datasets (see $[8,10,13]$ and $[14])$.

\section{Appendix}

\section{Linear Expansion of EEDL Distribution}

Let $w=\left\{1-\left[\left(1+\phi x^{-u}\right)^{-q}\right]\right\}^{-(1 / \gamma)}-1$

$$
\begin{aligned}
f_{X}(x) & =\alpha \beta \frac{e^{-\beta w}\left(1-e^{-\beta w}\right)^{\alpha-1}}{(1+w)^{-(\gamma+1)}} \\
f_{X}(x) & =\alpha \beta e^{-\beta w}\left(1-e^{-\beta w}\right)^{\alpha-1}(1+w)^{(\gamma+1)}, \\
f_{X}(x) & =\alpha \beta \sum_{i=0}^{\gamma+1} \frac{w^{i}}{\Gamma(\gamma-i+2)} e^{-\beta w}\left(1-e^{-\beta w}\right)^{\alpha-1}, \\
f_{X}(x) & =\alpha \beta \sum_{i=0}^{\gamma+1} \sum_{m=0}^{\alpha-1}(-1)^{m} \frac{w^{i} \Gamma(\alpha)}{\Gamma(\gamma-i+2) \Gamma(\alpha-m) \Gamma(m+1)} e^{-\beta w}, \\
w^{i} & =\sum_{j, k, l=0}^{\infty} \phi^{l} x^{-u l}(-1)^{i+l-j} \frac{\Gamma(j / \gamma+k) \Gamma(n+l)}{\Gamma(k+l) \Gamma(j / \gamma) \Gamma(l+1) \Gamma(n)} \\
f_{X}(x) & =\Omega \alpha \beta x^{-u l} e^{-\beta w}\left(1-e^{-\beta w}\right)^{\alpha-1},
\end{aligned}
$$

where

$$
\begin{aligned}
\Omega & =\sum_{i, j, k, l=0}^{\infty} \phi^{l}(-1)^{i+l-j} \frac{\Gamma(i+1) \Gamma(n+l) \Gamma(j / \gamma+k)}{\Gamma(r-i+2) \Gamma(i-j+1) \Gamma(k+1) \Gamma(j+1) \Gamma(j / \gamma) \Gamma(l+1) \Gamma(n)}, \\
f_{X}(x) & =\alpha \beta \Gamma(\alpha) \sum_{i, j, k, l, m=0}^{\infty} \frac{(-1)^{m} \Gamma(j / \gamma+k) \Gamma(n+l) \phi^{l}(-1)^{i+l-j}}{\Gamma(\gamma-i+2) \Gamma(\alpha-m) \Gamma(m+1) \Gamma(k+1) \Gamma(j / \gamma) \Gamma(l+1) \Gamma(n)} x^{-u l} e^{-\beta w(m+1)}, \\
f_{X}(x) & =\alpha \beta \frac{e^{-\beta w}\left(1-e^{-\beta w}\right)^{\alpha-1}}{(1+w)^{-(\gamma+1)}}, \\
f_{X}(x) & =\alpha \beta e^{-\beta w}\left(1-e^{-\beta w}\right)^{\alpha-1}(1+w)^{(\gamma+1)} .
\end{aligned}
$$




\section{Data Availability}

The two datasets used are appliances data and yarn data. Both datasets are provided in the body of the article.

\section{Conflicts of Interest}

The authors declare that they have no conflicts of interest.

\section{References}

[1] H. M. Yousof, A. Z. Afify, A. E. H. N. Ebraheim, G. G. Hamedani, and N. S. Butt, "On six-parameter frechet distribution: properties and applications," Pakistan Journal of Statistics and Operation Research, vol. 12, no. 2, pp. 281-299, 2016.

[2] C. Kleiber and S. Kotz, Statistical Size Distributions in Economics and Actuarial Sciences, Wiley, Hoboken, NJ, USA, 2003.

[3] C. Kleiber, A Guide to the Dagum Distributions, Springer, Berlin, Germany, 2007.

[4] R. Bandourian, R. Turley, and J. McDonald, "A comparison of parametric models for income distribution across countries and over time," SSRN Electronic Journal, vol. 305, 2002.

[5] F. Domma and F. Condino, "The beta-dagum distribution: definition and properties," Communications in StatisticsTheory and Methods, vol. 42, no. 22, pp. 4070-4090, 2013.

[6] B. O. Oluyede and S. Rajasooriya, "The mc-dagum distribution and its statistical properties with applications," Asian Journal of Mathematics and Applications, vol. 2013, Article ID ama0085, 2013.

[7] B. O. Oluyede and Y. Ye, "Weighted Dagum and related distributions," Afrika Matematika, vol. 25, no. 4, pp. 11251141, 2013.

[8] B. O. Oluyede, S. Huang, and M. Pararai, "A new class of generalized dagum distribution with applications to income and lifetime data," Journal of Statistical and Econometric Methods, vol. 3, no. 2, pp. 125-151, 2014.

[9] S. Huang and B. O. Oluyede, "Exponentiated KumaraswamyDagum distribution with applications to income and lifetime data," Journal of Statistical Distributions and Applications, vol. 1, no. 1, p. 8, 2014.

[10] A. O. Silva, L. C. Silva, and G. M. Cordeiro, "The extended dagum distribution: properties and application," Journal of Data Science, vol. 13, pp. 53-72, 2015.

[11] M. N. Shahzad and Z. Asghar, "Transmuted Dagum distribution: a more flexible and broad shaped hazard function model," Hacettepe Journal of Mathematics and Statistics, vol. 45, no. 52, p. 1, 2015.

[12] B. O. Oluyede, G. Motsewabagale, S. Huang, and S. Pararai, "The Dagum-Poisson distribution: model, properties and application," Electronic Journal of Applied Statistical Analysis, vol. 9, no. 1, pp. 169-197, 2016.

[13] S. Nasiru, P. N. Mwita, and O. Ngesa, "Exponentiated generalized exponential Dagum distribution," Journal of King Saud University-Science Direct, vol. 31, no. 3, pp. 362-371, 2017.

[14] H. Bakouch, M. Khan, T. Hussain, and C. Chesneau, "A power log-dagum distribution: estimation and applications," Journal of Applied Statistics, vol. 46, no. 5, pp. 874-892, 2018.

[15] N. L. Johnson, S. Kotz, and N. Balakrishnan, Continuous Univariate Distributions, Vol. 1, Wiley, Hoboken, NJ, USA, 2nd edition, 1994.
[16] M. A. Aljarrah, C. Lee, and F. Famoye, "On generating T-X family of distributions using quantile functions," Journal of Statistical Distributions and Applications, vol. 1, no. 2, 2014.

[17] P. F. Paranaíba, E. M. M. Ortega, G. M. Cordeiro, and R. R. Pescim, "The beta burr XII distribution with application to lifetime data," Computational Statistics \& Data Analysis, vol. 55, no. 2, pp. 1118-1136, 2011.

[18] G. M. Cordeiro and A. J. Lemonte, "The beta-half-cauchy distribution," Journal of Probability and Statistics, vol. 2011, pp. 1-18, 2011.

[19] A. Alzaatreh, C. Lee, and F. Famoye, "T-normal family of distributions: a new approach to generalize the normal distribution," Journal of Statistical Distributions and Applications, vol. 1, no. 1, p. 16, 2014.

[20] A. Alzaatreh, C. Lee, F. Famoye, and I. Ghosh, "The generalized cauchy family of distributions with applications," Journal of Statistical Distributions and Applications, vol. 3, no. 1, p. 16, 2016.

[21] M. Butt, A. Alzaatreh, G. Cordeiro, M. H. Tahir, and M. Mansoor, "On generalized classes of exponential distribution using $T$ - $X$ family framework," Filomat, vol. 32, no. 4, pp. 1259-1272, 2018.

[22] F. Famoye, E. Akarawak, and M. Ekum, "Weibull-normal distribution and its applications," Journal of Statistical Theory and Applications, vol. 17, no. 4, pp. 719-727, 2018.

[23] N. Eugene, C. Lee, and F. Famoye, "Beta-normal distribution and its applications," Communications in Statistics-Theory and Methods, vol. 31, no. 4, pp. 497-512, 2002.

[24] A. Alzaatreh, C. Lee, and F. Famoye, "A new method for generating families of continuous distributions," METRON, vol. 71, no. 1, pp. 63-79, 2013.

[25] M. A. Nasir, M. Aljarrah, F. Jamal, and M. H. Tahir, "A new generalized Burr family of distributions based on quantile function," Journal of Statistics Applications and Probability, vol. 6, no. 3, pp. 1-14, 2017.

[26] M. A. Nasir, M. H. Tahir, F. Jamal, and G. Ozel, "A new generalized burr family of distributions for the lifetime data," Journal of Statistics Applications and Probability, vol. 6, no. 2, pp. 4001-4017, 2017.

[27] F. Oluyede, M. A. Aljarrah, M. H. Tahir, and M. A. Nasir, "A new extended generalized burr-III family of distributions," Tbilisi Mathematical Journal, vol. 11, no. 1, pp. 59-78, 2018.

[28] F. Jamal, M. A. Nasir, M. H. Tahir, and N. H. Montazeri, "The odd Burr-III family of distributions," Journal of Statistics Applications \& Probability, vol. 6, no. 1, pp. 105-122, 2017.

[29] F. Jamal and M. A. Nasir, "Some new members of the $T-X$ family of distributions," in Proceedings of the 17th International Conference on Statistical Sciences, vol. 33, Lahore, Pakistan, January 2019.

[30] R. D. Gupta and D. Kundu, "Exponentiated exponential family: an alternative to gamma and weibull distributions," Biometrical Journal, vol. 43, no. 1, pp. 117-130, 2001.

[31] C. E. Shannon, "A mathematical theory of communication," Bell System Technical Journal, vol. 27, no. 3, pp. 379-423, 1948.

[32] J. F. Lawless, Statistical Models and Methods for Lifetime Data, Wiley, Hoboken, NJ, USA, 1982.

[33] M. Pal and M. Tiensuwan, "The beta transmuted weibull distribution," Austrian Journal of Statistics, vol. 43, no. 2, pp. 133-149, 2014.

[34] C. P. Quesenberry and J. Kent, "Selecting among probability distributions used in reliability," Technometrics, vol. 24, no. 1, pp. 59-65, 1982. 